\title{
ANALYSIS TOOLS FOR NEXT-GENERATION HADRON SPECTROSCOPY EXPERIMENTS*
}
M. Battaglieri ${ }^{\mathrm{a}}$, B.J. Briscoe ${ }^{\mathrm{b}}$, A. Celentano $^{\mathrm{a}}$, S.-U. Chung $^{\mathrm{c}, \mathrm{d}, \mathrm{e}}$ A. D'Angelo ${ }^{\text {f }}$, R. De Vita ${ }^{\mathrm{a}}$, M. Döring ${ }^{\mathrm{b}}$, J.J. DudeK ${ }^{\mathrm{g}, \mathrm{h}}$, S. Eidelman ${ }^{\mathrm{i}}$ S. Fegan ${ }^{\mathrm{a}}$, J. Ferretti ${ }^{\mathrm{j}}$, A. Filippi ${ }^{\kappa}$, G. Fox ${ }^{\mathrm{k}}$, G. Galata ${ }^{\mathrm{a}}$
H. García-Tecocoatzi ${ }^{1, a}$, D.I. Glazier ${ }^{m}$, B. Grube ${ }^{\mathrm{c}}$, C. Hanhart ${ }^{\mathrm{n}, \mathrm{o}}$ M. Hoferichter ${ }^{\mathrm{p}, \mathrm{q}, \mathrm{r}}$, S.M. Hughes ${ }^{\mathrm{s}}$, D.G. Ireland ${ }^{\mathrm{m}}$, B. Ketzer ${ }^{\mathrm{t}}$, F.J. Klein ${ }^{\mathrm{u}}$

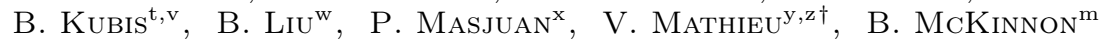 R. Mitchell ${ }^{y}$, F. Nerling ${ }^{\alpha}$, S. Paul ${ }^{\mathrm{c}}$, J.R. Peláez ${ }^{\beta}$, J. Rademacker ${ }^{\gamma}$ A. Rizzo ${ }^{f}$, C. Salgado ${ }^{\delta, g}$, E. Santopinto ${ }^{\mathrm{a}}$, A.V. Sarantsev $^{\mathrm{t}, \epsilon}$, T. Sato $^{\zeta}$ T. Schlüter ${ }^{\eta, \theta}$, M.L.L. Da Silva ${ }^{\iota}$, I. Stankovic ${ }^{\mathrm{s}}$, I. Strakovsky ${ }^{\mathrm{b}}$

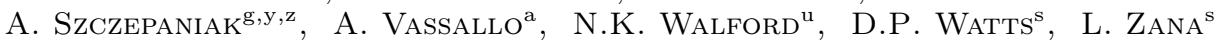

${ }^{a}$ INFN Sezione di Genova, Genova, Italy; ${ }^{b}$ The George Washington Univ., Washington D.C., USA; ${ }^{\mathrm{c}}$ Technische Univ. München, Physik Depart., Garching, Germany; ${ }^{\mathrm{d}}$ Depart. of Physics,

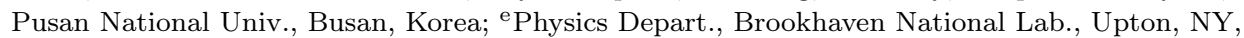
USA; ${ }^{\mathrm{f}}$ Univ. di Roma Tor Vergata and INFN, Sezione di Roma Tor Vergata, Rome, Italy; g Thomas Jefferson National Accelerator Facility, Newport News VA, USA; ${ }^{\mathrm{h}}$ Depart. of Physics, Old Dominion Univ., Norfolk, VA, USA; ${ }^{i}$ Budker Inst. of Nuclear Physics and Novosibirsk State Univ., Novosibirsk, Russia; ${ }^{j}$ Dipartimento di Fisica and INFN, Univ. di Roma Sapienza, Roma, Italy; ${ }^{\mathrm{k}}$ School of Informatics and Computing, Indiana Univ., Bloomington IN, USA; ${ }^{1}$ Inst. de Ciencias Nucleares, Univ. Nacional Autónoma de México, México DF, México; ${ }^{\mathrm{m}}$ SUPA, School of Physics and Astronomy, Univ. of Glasgow, UK; ${ }^{\mathrm{n}}$ Inst. für Kernphysik, Inst. for Advanced Simulations, Jülich, Germany; ${ }^{\circ}$ Jülich Center for Hadron Physics, Forschungszentrum Jülich, Jülich, Germany; PAlbert Einstein Center for Fundamental Physics, Inst. for Theor. Physics,

Univ. of Bern, Bern, Switzerland; ${ }^{9}$ Inst. für Kernphysik, Technische Univ. Darmstadt,

Darmstadt, Germany; ${ }^{\mathrm{r}}$ ExtreMe Matter Inst. EMMI, GSI Helmholtzzentrum für

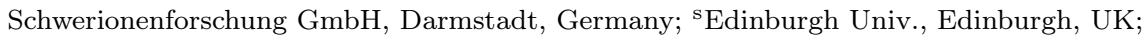
${ }^{t}$ Helmholtz-Inst. für Strahlen- und Kernphysik, Rheinische Friedrich-Wilhelms-Univ. Bonn, Bonn, Germany; ${ }^{\mathrm{u}}$ The Catholic Univ. of America, Washington D.C., USA; ${ }^{\mathrm{v}}$ Bethe Center for Theor. Physics, Univ. Bonn, Bonn, Germany; ${ }^{w}$ Inst. of High Energy Physics, Beijing, China; ${ }^{x}$ PRISMA Cluster of Excellence and Inst. für Kernphysik, Johannes Gutenberg-Univ. Mainz,

Mainz, Germany; ${ }^{y}$ Depart. of Physics, Indiana Univ., Bloomington IN, USA; ${ }^{\mathrm{z} C e n t e r ~ f o r ~}$

Exploration of Energy and Matter, Indiana Univ., Bloomington IN, USA; ${ }^{\alpha}$ Helmholtz-Inst. Mainz, GSI Helmholtzzentrum Darmstadt, Darmstadt, Germany; ${ }^{\beta}$ Depart. de Física Teórica II,

Univ. Complutense, Madrid, Spain; ${ }^{\gamma}$ H.H. Wills Physics Lab, Univ. of Bristol, Bristol, UK;

${ }^{\delta}$ Norfolk State Univ., Norfolk VA, USA; ${ }^{\epsilon}$ Petersburg Nuclear Physics Inst., Gatchina, Russia;

$\zeta$ Depart. of Physics, Osaka Univ., Toyonaka, Osaka, Japan; ${ }^{\eta}$ Ludwig-Maximilians-Univ., München, Germany; ${ }^{\theta}$ Excellence Cluster Universe, Technische Univ. München, Garching, Germany; ${ }^{\iota}$ Inst. de Fisica e Matematica, Univ. Federal de Pelotas, Pelotas, RS, Brazil; ${ }^{\kappa}$ INFN Sezione di Torino, Torino, Italy

(Received January 5, 2014)

\footnotetext{
${ }^{*}$ Funded by $\mathrm{SCOAP}^{3}$ under Creative Commons License, CC-BY 3.0.

$\dagger$ Corresponding author, Vincent.MATHIEU@umons.ac.be.
} 
The series of workshops on New Partial-Wave Analysis Tools for NextGeneration Hadron Spectroscopy Experiments was initiated with the ATHOS 2012 meeting, which took place in Camogli, Italy, June 20-22, 2012. It was followed by ATHOS 2013 in Kloster Seeon near Munich, Germany, May 21-24, 2013. The third, ATHOS3, meeting is planned for April 13-17, 2015 at The George Washington University Virginia Science and Technology Campus, USA. The workshops focus on the development of amplitude analysis tools for meson and baryon spectroscopy, and complement other programs in hadron spectroscopy organized in the recent past including the INT-JLab Workshop on Hadron Spectroscopy in Seattle in 2009, the International Workshop on Amplitude Analysis in Hadron Spectroscopy at the ECT*-Trento in 2011, the School on Amplitude Analysis in Modern Physics in Bad Honnef in 2011, the Jefferson Lab Advanced Study Institute Summer School in 2012, and the School on Concepts of Modern Amplitude Analysis Techniques in Flecken-Zechlin near Berlin in September 2013. The aim of this document is to summarize the discussions that took place at the ATHOS 2012 and ATHOS 2013 meetings. We do not attempt a comprehensive review of the field of amplitude analysis, but offer a collection of thoughts that we hope may lay the ground for such a document. The material presented in the article was edited by the following Editorial Board: Marco Battaglieri, Bill J. Briscoe, Su-Urk Chung, Michael Döring, Józef Dudek, Geoffrey Fox, Christoph Hanhart, Martin Hoferichter, David G. Ireland, Bernhard Ketzer, Bastian Kubis, Vincent Mathieu, Ryan Mitchell, José R. Peláez, Elena Santopinto, Adam Szczepaniak.

DOI:10.5506/APhysPolB.46.257

PACS numbers: 13.25.-k, 13.30.-a, 14.20.-c, 14.40.-n

\section{Introduction}

[C. Hanhart, M. Hoferichter, B. Kubis]

Quantum Chromodynamics (QCD), the fundamental theory of the strong interactions, defines the interactions of quarks and gluons, both types carrying the so-called color charge, which form the fundamental constituents of hadrons ${ }^{1}$. At high energies, these partons become asymptotically free, and systematic calculations based on perturbation theory in the strong coupling constant are possible and extremely successful. However, especially inside light hadrons that are in the focus of this manuscript, the average energies and momenta of partons are below the scale at which perturbation theory can be justified, and hadron properties are determined by interactions that are genuinely non-perturbative in nature. In particular, the bulk of hadron masses originates from gluonic self-interactions, which lead to forces

\footnotetext{
${ }^{1}$ All composite objects of quarks and gluons that are, therefore, subject to the strong interaction with no net color charge are called hadrons.
} 
that bind the constituents within distances smaller than $10^{-15} \mathrm{~m}$ in a way that only allows objects neutral with respect to the color charge to exist as physical, asymptotic states - a phenomenon known as confinement. As a consequence, the elementary degrees of freedom of the underlying theory only manifest themselves indirectly in the physical spectrum, which instead is built from composite, colorless hadrons. Just as atomic spectroscopy was instrumental in elucidating the underlying electromagnetic interactions, hadron spectroscopy is, therefore, the foremost laboratory for studying the implications of QCD.

While for many years the quark model has provided the main template for the spectrum of hadrons, recent developments in lattice simulations on the one side and effective-field-theory methods on the other have opened new avenues for investigations of hadron properties that are rooted in QCD. One of the most mysterious parts of the spectrum concerns the phenomenology of low-energy gluons and thus a complete mapping of gluonic excitations - that may manifest themselves either in hybrid states (states with both quarks and gluons as active, valence degrees of freedom) or in glueballs (states formed from gluons only) — is a central part of the present and future investigations of the hadron spectrum.

The anticipated accuracy of the next-generation hadron spectroscopy experiments will, in principle, allow for the identification of hadronic resonances for which either a reliable determination of their resonance parameters has proven elusive or even their very existence could not be unambiguously established before. Frequently, their identification is complicated by the occurrence of overlapping resonances, pole positions far in the complex plane, or weak couplings to the channels experimentally accessible. The main challenges include the development of parameterizations and their incorporation into partial-waves analyses that respect the theoretical constraints and allow for a reaction-independent determination of pole positions and residues, which uniquely characterize the properties of a given resonance. In this paper, we review some aspects of the theoretical and phenomenological underpinning of experimental data analyses that aim at extracting hadron resonance parameters in a controlled way.

Beyond providing a deeper understanding of the inner workings of $\mathrm{QCD}$, a theoretical control over hadronic final-state interactions is also essential to employ the decays of heavy mesons for the hunt of physics beyond the Standard Model of particle physics (SM), which are driven by the electroweak interactions: in order to explain the matter-antimatter asymmetry of the universe, an amount of $\mathrm{CP}$ violation is necessary that exceeds that of the SM by many orders of magnitude. Thus, additional CP violation has to be present, and it has to exceed the SM predictions dramatically. 
If present, $\mathrm{CP}$ violation in the decay of heavy mesons will show up as a complex phase, and, therefore, relies on interference of different amplitudes. As the observation of $\mathrm{CP}$ asymmetries in (partial) decay rates depends on both weak and strong phase differences, a more accurate understanding of the latter necessarily leads to an improved determination of the former, and resonating strong final states provide ideal enhancement factors for (probably very small) weak asymmetries. Therefore, the decay of a heavy meson into three or more light mesons appears to provide an ideal environment for $\mathrm{CP}$ studies due to the presence of a large number of meson resonances in the phase space available. Furthermore, besides enhancing the CP signals, the non-trivial distribution of the strong phase motion over the Dalitz plot allows for a test of systematics, and provides some sensitivity to the operator structure of the CP-violating source underlying the transition.

This twofold perspective of amplitude analyses should be kept in mind throughout this document: while a strong motivation clearly consists in understanding the spectrum of QCD as such, there is a strong benefit from making the results available for communities more concerned with the investigation of electroweak interactions and New Physics searches in hadronic environments.

\subsection{Quark Model}

\section{[V. Mathieu]}

The Quark Model was originally introduced as a classification scheme to organize the hadron spectrum. Since its introduction, significant progress has been made in the understanding of $\mathrm{QCD}$, and while there is no formal relation between constituent quarks and the QCD degrees of freedom, the lattice QCD hadron spectrum closely resembles that of the quark model. In the quark model, mesons are bound states of a valence, constituent quark and antiquark, while baryons contain three quarks. Quantum numbers of quark model bound states are obtained by combining the quantum numbers of the individual quark constituents, e.g. their spins and angular momenta. For example, the meson spin $J$ is given by the vector sum of quark-antiquark spin $s$ and orbital angular momentum $l$. Meson parity $P$ and, for neutral states, charge conjugation $C$ are given by $P=(-1)^{l+1}, C=(-1)^{l+s}$, respectively. It thus follows that certain combinations of total spin $J^{P C}, 0^{--}, 0^{+-}$, $1^{-+}, 2^{+-}, 3^{-+}, \ldots$, do not correspond to a quark-antiquark pair. These are referred to as exotic. There are no exotic baryons in a corresponding sense, i.e. three quarks can be combined to give any combination of a half-integer spin and parity. In addition, taking into account quark flavors, the quark model arranges hadrons into flavor multiplets with mass degeneracies broken by the quark masses. 
The classification of the well-established light mesons according to the quark model is summarized in Table I taken from the Review of Particle Physics [1]. Indeed, most of the observed resonances fit into the quarkmodel pattern, although several states including the $\rho_{2}$ or the $b_{3}$ are missing. There are also well-established resonances that do not fit the quark-model classification. These include, for example, states with $J^{P C}=0^{++}$quantum numbers, such as the $f_{0}(500)$.

Hadron resonances can also be classified by the Regge trajectories they belong to. For example, for mesons, Regge trajectories are labeled by signature $\tau=(-1)^{J}$, naturality $\eta=P(-1)^{J}$, and also by isospin $I$ and $G$-parity $G=C(-1)^{I}$.

TABLE I

Well-established mesons classified according to the Quark Model.

\begin{tabular}{cccccc}
\hline \hline$n^{2 s+1} \ell_{J}$ & $J^{P C}$ & $I=1$ & $I=1 / 2$ & $I=0$ & $I=0$ \\
\hline $1^{1} S_{0}$ & $0^{-+}$ & $\pi$ & $K$ & $\eta$ & $\eta^{\prime}$ \\
$1^{3} S_{0}$ & $1^{--}$ & $\rho(770)$ & $K^{*}(892)$ & $\omega(782)$ & $\phi(1020)$ \\
\hline $1^{1} P_{1}$ & $1^{+-}$ & $b_{1}(1235)$ & $K_{1}(1400)$ & $h_{1}(1170)$ & $h_{1}(1380)$ \\
$1^{3} P_{0}$ & $0^{++}$ & $a_{0}(1450)$ & $K_{0}^{*}(1430)$ & $f_{0}(1370)$ & $f_{0}(1710)$ \\
$1^{3} P_{1}$ & $1^{++}$ & $a_{1}(1260)$ & $K_{1}(1270)$ & $f_{1}(1285)$ & $f_{1}(1420)$ \\
$1^{3} P_{2}$ & $2^{++}$ & $a_{2}(1320)$ & $K_{2}^{*}(1430)$ & $f_{2}(1270)$ & $f_{2}^{\prime}(1525)$ \\
\hline $1^{1} D_{2}$ & $2^{-+}$ & $\pi_{2}(1670)$ & $K_{2}(1770)$ & $\eta_{2}(1645)$ & $\eta_{2}(1870)$ \\
$1^{3} D_{1}$ & $1^{--}$ & $\rho(1700)$ & $K^{*}(1680)$ & $\omega(1650)$ & \\
$1^{3} D_{2}$ & $2^{--}$ & & $K_{2}(1820)$ & & \\
$1^{3} D_{3}$ & $3^{--}$ & $\rho_{3}(1690)$ & $K_{3}^{*}(1780)$ & $\omega_{3}(1670)$ & $\phi_{3}(1850)$ \\
\hline $1^{1} F_{3}$ & $3^{+-}$ & & & & \\
$1^{3} F_{2}$ & $2^{++}$ & & $K_{2}^{*}(1980)$ & $f_{2}(1910)$ & $f_{2}(2010)$ \\
$1^{3} F_{3}$ & $3^{++}$ & & $K_{3}(2320)$ & & \\
$1^{3} F_{4}$ & $4^{++}$ & $a_{4}(2040)$ & $K_{4}^{*}(2045)$ & & $f_{4}(2050)$
\end{tabular}

\subsection{Lattice $Q C D$ and the hadron spectrum}

\section{[J.J. Dudek, M. Döring]}

Lattice QCD is a first principles numerical approach to QCD which considers the field theory evaluated on a finite grid of points. Supercomputers are used to Monte Carlo sample a finite, but large, number of gluon field configurations according to their importance in the QCD Euclidean path integral. Color-singlet correlation functions can then be computed using this ensemble of configurations, with the mean and variance over the ensemble providing an estimate and an uncertainty. The discrete spectrum of eigenstates of the theory can be extracted from the time-dependence of correlation functions. 
In principle, this is a systematically improvable approach to QCD. Calculations can be performed for a range of lattice spacings, $a$, and an extrapolation $a \rightarrow 0$ performed. Similarly, the behavior with increasing finite volume can be studied. In practice, the low mass of the physical $u$ and $d$ quarks provides a challenge - the numerical algorithms used to generate gluon field configurations and to compute quark propagation scale badly with decreasing quark mass. Furthermore, since very light quarks imply very light pions with large Compton wavelengths, there is a need to increase the size of the lattice volume as the quark mass decreases. For fixed lattice spacing, this requires more points in the grid and thus increased computation time.

For relatively simple quantities like the masses of the lightest stable hadrons, precision calculations considering all the above systematic variations have recently been carried out. An example is presented in Fig. 1. In the case of excited hadrons, the state of the art is not yet at this level,

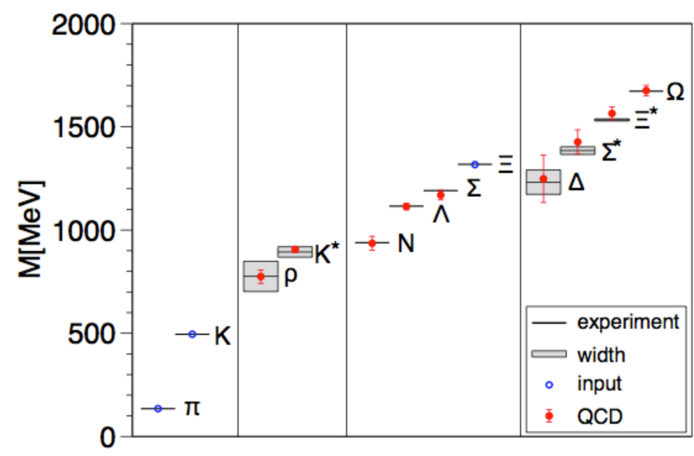

Fig. 1. The light hadron spectrum of QCD computed using lattice techniques in [5].

with calculations typically being performed at a single (albeit small) lattice spacing, and with light quark masses chosen to be somewhat above the physical value. Figure 2 presents an example of recent progress in determining the excited isoscalar and isovector meson spectrum. This calculation has approximately physical strange quarks but light quarks somewhat heavier than physical such that the pion has a mass of $391 \mathrm{MeV}[2-4]$.

Figure 2 shows a detailed spectrum of excited states of various $J^{P C}$, with many of the observed experimental systematics being reproduced, as well as those of the $n^{2 S+1} L_{J} q \bar{q}$ quark model. A clear set of exotic $J^{P C}$ states are extracted with the isovector spectrum featuring a lightest $1^{-+}$roughly $1.3 \mathrm{GeV}$ heavier than the $\rho$ meson. Slightly heavier than the $1^{-+}$is a single $0^{+-}$state and two $2^{+-}$states, and these observations have been shown to be robust with increasing quark mass. Examination of the type of quark-gluon operator constructions which have large overlap with these exotic states suggests that they are hybrid mesons with $q \bar{q}$ in a color octet coupled to a chromomagnetic gluonic excitation. Such a construction can also generate 


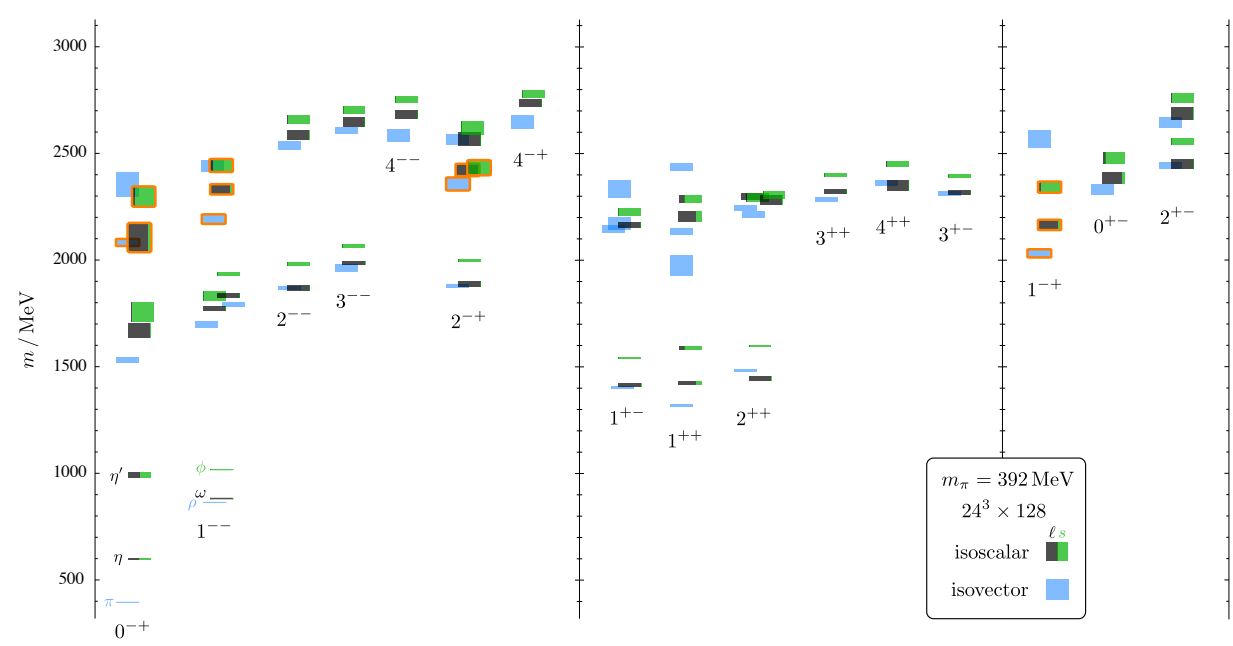

Fig. 2. (Color online) Isoscalar and isovector meson spectrum determined in a lattice QCD calculation with $m_{\pi}=391 \mathrm{MeV}$ [2].

non-exotic hybrid mesons, and, indeed, such states with $J^{P C}=0^{-+}, 2^{-+}$, and $1^{--}$are identified in the calculation (highlighted in orange in Fig. 2). Calculation in the charmonium sector $[6,7]$ shows similar conventional meson and hybrid-meson systematics.

The baryon spectrum has been computed using related techniques [8, 9], see also Refs. [10-13]. Hybrid baryons, which cannot have exotic quantum numbers, have been predicted [14] with a quantum number distribution and operator overlaps that suggest the same chromomagnetic gluonic excitation is at work.

Computing the spectrum of glueballs is relatively straightforward within the pure-glue theory where the existence of quarks is ignored. Glueball operators can be constructed out of gluon fields and the spectrum extracted from correlation functions. The spectra so determined in [15, 16] show that the lightest glueballs have non-exotic $J^{P C}$ with a lightest $0^{++}$, and somewhat heavier a $2^{++}$and a $0^{-+}$. However in QCD, with quarks, these glueball basis states should appear embedded within a spectrum of isoscalar mesons, possibly strongly mixed with $q \bar{q}$ basis states. Such calculations have proven to be very challenging, for example the calculation in [2] was not able to observe any states having strong overlap with glueball operators, which produced statistically noisy correlation functions. In short, the role of glueballs in the meson spectrum has not been determined in lattice QCD.

Returning to Fig. 2, although a lot of the correct physics is present, including annihilation of $q \bar{q}$ pairs and the corresponding mixing of hidden-light and hidden-strange configurations, the calculations are clearly not complete. Most of the states extracted should, in fact, be unstable resonances decaying 
into multi-meson final states. In fact, within a finite-volume theory, there cannot be a continuum of multi-meson states, rather there must be a discrete spectrum and the volume-dependence of this spectrum can be mapped onto hadron scattering amplitudes [17-26] — often calling for the inclusion of inelasticities [23, 27-31]. The full richness of this spectrum was not resolved in [2] as only quasi-local $q \bar{q}$-like operator constructions were used, and these have very poor overlap onto multi-meson states.

The current frontier in lattice QCD calculations of hadron spectroscopy involves the inclusion of operators that efficiently interpolate multi-meson states and the extraction of the complete discrete spectrum of states in a finite volume. An example of what can currently be achieved is presented in Fig. 3. By computing the complete low-energy spectrum of states with isospin 1 in multiple finite volumes, and applying the finite-volume formalism $[17,18]$ to determine the elastic $P$-wave scattering phase shift, a rapid rise characteristic of a resonance can be observed. Fitting the phase shift with a simple Breit-Wigner form yields an estimate of the $\rho$ resonance mass and width in a version of QCD where the pion mass is $391 \mathrm{MeV}$ [32]. See Refs. [21, 22, 33-36] for other studies on the $\rho$ decay using the Lüscher formalism. Ongoing calculations are addressing higher resonances which can decay into multiple channels. The first coupled-channel lattice QCD calculation has been completed recently [26]. Concepts are developed to deal with three-body and higher scattering in the finite volume [37-40].

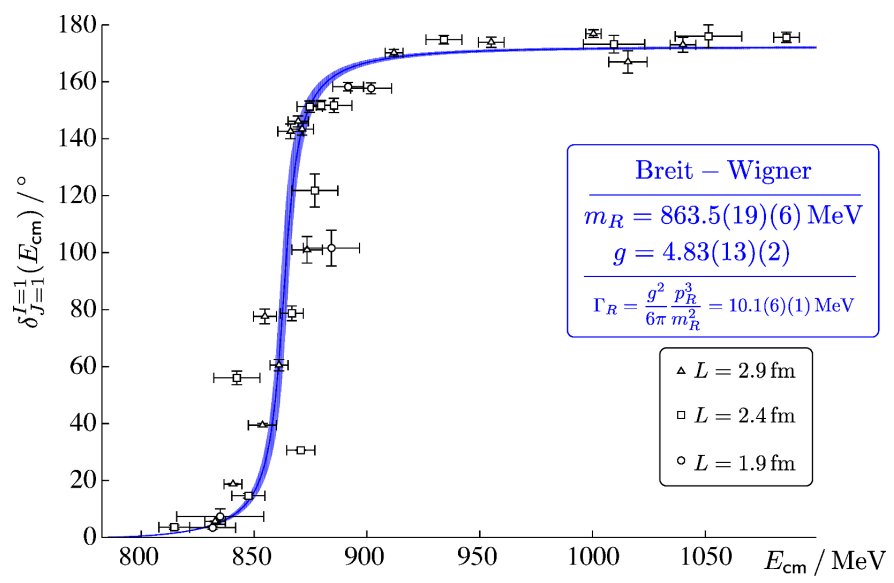

Fig. 3. The isospin $1, P$-wave $\pi \pi$ scattering phase shift determined from the discrete spectrum in three different lattice volumes. Calculation performed with quark masses such that $m_{\pi}=391 \mathrm{MeV}$ [32].

In the near future, we envisage the possibility of using the same scattering amplitude parameterizations to describe experimental data and the finitevolume spectra of QCD computed using lattice techniques. 


\section{Experiments}

[M. Battaglieri, D.G. Ireland, B. Ketzer, R. Mitchell, A.V. Sarantsev]

A number of hadron spectroscopy experiments are currently in operation, and several new ones are expected to come online in the near future. At low energies, fixed-target experiments studying elastic or quasi-elastic mesonnucleon scattering reactions have been instrumental for baryon spectroscopy. Most of our information about $N^{*}$ and $\Delta$ resonances stems from direct production in elastic and inelastic $\pi N$ scattering experiments from more than 30 years ago. In these reactions, also referred to as $s$-channel production or formation, the beam and the target merge to produce the resonance, which then subsequently decays. Phase-shift analysis in the elastic region is a well-defined procedure that yields the scattering amplitude from the experimental data with only a few discrete alternative solutions. Elastic pion-nucleon scattering still provides the foundation for precise partial-wave analyses of baryon resonances, but an improvement to the data set of mesoninduced reactions is only likely to be possible with the use of secondary hadron beams, produced at future facilities such as J-PARC or an electronion collider (EIC).

Current fixed-target experiments at electron machines like CLAS (JLAB), A2 (MAMI), or CBELSA/TAPS (ELSA) mainly use photoproduction of resonances on proton targets to study baryon excitations. The JLab12 upgrade, with the two new detectors GlueX and CLAS12, will enable a dedicated program of spectroscopy in which a major goal will be the discovery of hybrid mesons and baryons containing light quarks.

High-energy fixed-target experiments with hadron beams like VES (IHEP Protvino) or COMPASS (CERN) study $t$-channel reactions of the beam particles with protons or nuclear targets. The target particle merely serves as a strong-interaction partner and takes up the recoil.

Experiments with hadrons containing charm or bottom quarks require higher center-of-mass energies, and are performed either at $e^{+} e^{-}$colliders, such as BES-III (BEPC), KEDR (VEPP-4M), and Belle-II (KEKB), at $p p$ colliders such as ALICE, ATLAS, CMS, and LHCb (LHC), or using $p \bar{p}$ annihilations like at the future PANDA experiment at the High-Energy Storage Ring (HESR) for antiprotons at FAIR.

Key ingredients for next-generation experiments in hadron spectroscopy are:

- detectors for both charged and neutral particles, with excellent resolution and particle identification capability;

- beam energies high enough to ensure sufficient phase space for production; 
- high statistics, for sensitivity to production cross sections at picobarn level;

- networks of experimentalists and theorists working together for the development of common analysis tools.

\subsection{Fixed-target experiments}

Fixed-target experiments with primary electron or proton beams as well as secondary meson $(\pi, K)$ or photon beams have been at the heart of mapping out and understanding the light hadron spectrum for more than 30 years.

Depending on the incident particle, the energy and the final-state kinematics, different mechanisms contribute to the production of excited meson states: $s$-channel resonance formation at low energies, and production reactions involving a recoil particle at higher energies. At very high energies, $t$-channel reactions like diffraction, central production, or photoproduction involving quasireal photons dominate.

\subsubsection{Hadron beams: COMPASS, VES, and PANDA}

COMPASS [41, 42] is a high-energy hadron physics experiment at the Super Proton Synchrotron at CERN involving about 220 physicists from 13 countries and 24 institutions. One of the purposes of this experiment is to study hadron spectroscopy using high-intensity hadron beams of 150 $250 \mathrm{GeV}$ by diffractive, central, and Coulomb production reactions. Final states containing charged and neutral particles are detected with high resolution over a wide angular range, provided by a two-stage magnetic spectrometer equipped with precision vertex detectors, charged-particle tracking, particle identification, and calorimetry. A uniform acceptance for both charged and neutral particles as realized in COMPASS is mandatory for a reliable partial-wave analysis.

One of the goals of COMPASS is to understand and map out the spectrum of mesons up to masses of about $2.5 \mathrm{GeV}$ with high statistical accuracy, and to look for possible signatures of states which cannot be explained within the constituent quark model, e.g. multi-quark and hybrid states or glueballs. The non- $q \bar{q}^{\prime}$ nature of a resonance may be identified either through exotic quantum numbers, which require additional contributions beyond a quark and an antiquark, or via an overpopulation of states compared to expectations from the quark model. The latter approach, however, requires the unambiguous identification of all states of a given $J^{P C}$ nonet, a task which has been achieved so far only for the $L=0,1$ mesons, $c f$. Table I.

In a first analysis of the $\pi^{-} \pi^{-} \pi^{+}$final state from the scattering of $190 \mathrm{GeV} \pi^{-}$on a $\mathrm{Pb}$ target, recorded in 2004 , a clear signal in intensity and phase motion in the $J^{P C}=1^{-+} I^{G}=1^{+} \rho \pi P$ partial wave has been 
observed by COMPASS [43], consistent with the $\pi_{1}(1600)$. However, a large background, possibly due to Deck-like processes, is also present in the data, and will have to be properly taken into account in a more refined partialwave analysis. Two orders of magnitude more data with pion and proton beams on proton and nuclear targets have been collected in 2008, 2009, and 2012 , recording samples of various final states: $54 \mathrm{M}$ events for $3 \pi, 144 \mathrm{k}$ events for $K \bar{K} \pi \pi, 116 \mathrm{k}$ events for $\eta \pi, 39 \mathrm{k}$ events for $\eta^{\prime} \pi$, etc. A signal for an exotic $1^{-+}$state is also observed in the $\eta^{\prime} \pi$ final state [44]. As for the $3 \pi$ final state, however, the clear distinction between resonant and non-resonant contributions requires a more reliable model for the background processes to be included in the fit to the spin-density matrix. The new data for the first time allow an analysis in narrow bins of invariant mass and 4-momentum transfer $t$, which has the power to shed more light on the relative contribution of resonant and non-resonant processes in this and other waves, e.g. the well-known $a_{1}(1260)$.

The VES experiment uses a $28 \mathrm{GeV}$ secondary pion beam from the U-70 proton synchrotron at IHEP Protvino incident on a Be target to study lightmeson resonances decaying to neutral and charged pions. The analysis techniques used are very similar to the ones employed at COMPASS.

PANDA is one of the major projects planned for the FAIR-Facility in Darmstadt. FAIR is an extension of the existing Heavy Ion Research Lab (GSI) and is expected to start operation in 2018. PANDA studies interactions between antiprotons and fixed target protons and nuclei in the momentum range of $1.5-15 \mathrm{GeV}$ using the high-energy storage ring HESR. The PANDA Collaboration, with more than 450 scientists from 17 European countries, intends to do basic research on various topics around the weak and strong forces, exotic states of matter, and the structure of hadrons.

\subsubsection{Electron beams: CLAS, ELSA, MAMI, SPring-8, and JLAB12}

In the last 20 years, electron accelerators such as CEBAF at JLab, ELSA at Bonn, MAMI at Mainz, and SPring-8 in Japan, have considerably improved in the delivery of electron and photon beams of high intensity and quality to enable coincidence measurements for hadron spectroscopy. New detectors and targets have been designed and commissioned. We are now in a situation where the photo- and electroproduction of pseudoscalar mesons carry the highest potential to investigate the baryonic spectrum. In addition to the resonance positions and strong residues, which describe couplings to decay channels, the electromagnetic couplings and transition form factors are also being investigated.

Pseudoscalar (e.g. $\pi, \eta, K$, and $\eta^{\prime}$ ) photoproduction is one of the cleanest ways to study direct baryon production. In fact, this reaction is described by a set of only four transition amplitudes [45] (invariant, spin, transversity, or 
helicity amplitudes). With the developments in beams and target mentioned above, we are now very near to being able to extract these amplitudes (up to an overall phase) from a combination of polarization experiments.

It is also very important that data obtained with a proton target are complemented with data from the neutron, albeit in quasi-free production from a nuclear target. At JLab, data taken with deuterium targets, in particular the HD-ICE target, will provide information on the $\gamma$-neutron couplings of excited states by extracting single- and double-polarization observables. Complementary campaigns at ELSA and MAMI will also provide important data sets.

Data obtained from the neutron bound in the nuclear targets (mostly deuteron) will be sensitive to nuclear effects such as Fermi motion and finalstate scattering. Great care needs to be taken in unfolding the desired amplitudes and multipoles. At present, the experimental information from proton reactions is substantially larger then that from (quasi-)neutron reactions (the $\gamma N \rightarrow \pi N$ database is just $15 \%$ of the proton database), and this difference is especially acute for polarized experiments. Only with sufficient data on both proton and neutron targets, can one hope to disentangle the isoscalar and isovector electromagnetic couplings of the various $N^{*}$ and $\Delta^{*}$ resonances, as well as the isospin properties of the non-resonant background amplitudes.

The search for mesons with exotic quantum numbers is the primary aim of the GlueX experiment at a future $12 \mathrm{GeV}$ upgrade of Jefferson Laboratory. The GlueX experiment will map out the meson spectrum with unprecedented statistics using photoproduction, a complementary reaction mechanism to others studied so far (which include hadroproduction with pion, kaon, or proton beams, or heavy meson decays). With $9 \mathrm{GeV}$ photons, the mass range extends up to $2.5-3 \mathrm{GeV}$ and will cover the region where the light exotic multiplet is expected. A complementary meson spectroscopy program will be carried out at Hall-B with the new CLAS12 detector. The technique, electroproduction at very low $Q^{2}\left(0.01-0.1 \mathrm{GeV}^{2}\right)$, provides a high photon flux as well as a high degree of linear polarization, and represents a competitive and complementary way to study the meson spectrum and production mechanisms with respect to real photoproduction experiments. After a calibration period, the detector will begin to record data in 2015/16. Both GlueX and CLAS12 physics programs will start in conjunction with the analysis of the golden channels $\eta \pi, \eta^{\prime} \pi$, and $3 \pi$ for the detection of hybrid mesons. A detailed theoretical study on these channels is then required in the near future for the success of these experiments. 


\subsection{Annihilation reactions: Belle-II, BES-III, CMD-3, LHCb, and SND}

Annihilation of $e^{+} e^{-}$and $p \bar{p}$ have been historically important additions to the host of reactions in hadron spectroscopy. The early experiments in the SLAC-LBL $e^{+} e^{-}$storage ring (SPEAR) produced many of the first measurements in the charmonium spectrum. They were followed by, among others, CLEO, BaBar, Belle, BES-III, CMD-3 and SND, with the latter three still in operation. Charmonium decay data sets have been supplemented by bottomonium decay data and open-flavor $D$ and $B$ meson decays. Proton-antiproton annihilation was studied at the Low Energy Antiproton Ring (LEAR) at CERN and new experiments at center-of-mass energies above charm threshold are planned for the FAIR facility (see the description of PANDA in the fixed target experiments section). LHCb is exploiting the highest energy ever reached by the LHC to produce a huge number of mesons and study their decays.

There has recently been a dramatic renewal of interest in the subjects studied by these experiments. This renaissance has been driven in part by experimental reports of $D^{0} \bar{D}^{0}$ mixing and the discovery of narrow $D_{s J}$ states and a plethora of charmonium-like $X Y Z$ states at the $B$ factories, as well as the observation of an intriguing proton-antiproton threshold enhancement and the possibly-related $X(1835)$ meson state at BES-II. Many of these studies have relied on amplitude analysis techniques and phenomenology. For example, during the $B$ factory age, the program to extract weak interaction parameters (such as the CKM matrix elements) or to study New Physics effects went through the analysis of decays with final states with at least three particles. Light hadron final-state interactions bring in phases, which interfere with the weak phases and have to be included in an amplitude analysis. The $D^{0} \rightarrow K_{s} \pi \pi$ amplitude, as one example, depends on the weak CKM phase $\gamma$, which can only be extracted if the strong $K \pi$ and $\pi \pi$ phases are known $[46,47]$.

Here, we briefly describe five facilities that are currently in operation or are planned. The BES-III experiment at BEPCII in Beijing [48], which started operation in the summer 2008, has accumulated data samples corresponding to 1.3 billion $J / \psi$ decays, 0.6 billion $\psi(3686)$ decays, $2.9 \mathrm{fb}^{-1}$ at the peak of the $\psi(3770)$ resonance, and around $4 \mathrm{fb}^{-1}$ above $4 \mathrm{GeV}$. These samples can be used for precision spectroscopy amplitude analysis. Coupled with the currently available results from CLEO-c, BES-III will make it possible to study in detail, and with unprecedented high precision, light hadron spectroscopy in the decays of charmonium states. In addition, about 90 million $D \bar{D}$ pairs will be collected at BES-III in a three-year run at the $\psi(3770)$ peak, which will allow many high precision measurements, including CKM matrix elements related to charm weak decays, decay constants $f_{D+}$ and $f_{D_{S}}$, Dalitz decays of three-body $D$ meson decays, searches for CP violation 
in the charmed-quark sector, and absolute decay branching fractions. With modern techniques and huge data samples, searches for rare, lepton-numberviolating, flavor-violating, and/or invisible decays of $D$ mesons, charmonium resonances, and $\tau$ leptons will be possible.

Since 2010, experiments have been in progress at the upgraded VEPP$2000 e^{+} e^{-}$collider operated in the center-of-mass energy range from the threshold of hadron production up to $2 \mathrm{GeV}$. Two detectors are used: CMD-3 and SND. The goal of the CMD-3 and SND experiments is to study the spectroscopy of the light vector mesons ( $\rho, \omega$, and $\phi$ and their excitations) and to measure the cross sections of various exclusive channels of $e^{+} e^{-}$ annihilation with high accuracy. Such measurements should help clarify the muon $g-2$ puzzle and provide opportunities for detailed studies of the dynamics of multi-hadron final states. The expected data samples should be sufficiently large to disentangle various intermediate mechanisms, such as those present in the first high-statistics studies of the four-pion final state in $e^{+} e^{-}$annihilation at CMD-2 [49,50] and $\tau$ decays at CLEO [51]. A crucial issue for a successful partial-wave analysis is to use full event information. With future running in the energy range from 1 to $2 \mathrm{GeV}$, one expects data samples of $10^{5}$ and larger for the dominant final states with three to six pions.

The VEPP-4M $e^{+} e^{-}$collider covers a center-of-mass energy range from $2 \mathrm{GeV}$ to $11 \mathrm{GeV}$. It is currently operated in the charmonium family range with the KEDR detector. Successful application of two methods for the high-precision determination of the absolute beam energy — resonant depolarization and Compton backscattering - resulted in various experiments with record accuracy. Among them are measurements of the $J / \psi$ and $\psi(2 S)$ masses [52]; of the total and leptonic width of the $J / \psi$ [53], $\psi(2 S)$ [54], and $\psi(3770)$ [55]; the $D^{0}$ and $D^{ \pm}$masses [56]; the $\tau$ lepton mass [57]; and a search for narrow resonances from $1.85 \mathrm{GeV}$ to the $J / \psi$ mass [58]. Also planned is a new measurement of $R$ up to $8 \mathrm{GeV}$.

The Belle-II experiment (KEK, Japan) will build off the enormous success of the previous BaBar (SLAC, USA) and Belle experiments. The primary goal will be to use $e^{+} e^{-}$collisions to produce correlated pairs of $B$ mesons through $\Upsilon(4 S)$ decays. As demonstrated at BaBar and Belle, these decays allow precision tests of the Standard Model. But, at the same time, decays of $B$ mesons have proven to be an efficient source of many of the still-unexplained $X Y Z$ states of charmonium. In addition, other techniques, such as initial-state radiation, in which the center of mass of the $e^{+} e^{-}$annihilation is lowered via the radiation of an initial-state photon, have also allowed for the discovery of other $X Y Z$ states, as well as the production of light-quark vector mesons. While BaBar and Belle accumulated of the order of $1.5 \mathrm{ab}^{-1}$ of data, Belle-II will use the upgraded collider at KEK to collect a projected $50 \mathrm{ab}^{-1}$ of $e^{+} e^{-}$data by 2020 . 
The LHCb experiment [59] is designed to exploit the huge $b \bar{b}$ cross section at $p p$ collisions at LHC energies [60] for precision flavor physics. The same characteristics that optimize LHCb for $b$ physics also make it an excellent charm physics experiment, benefiting from a charm cross section of $(6.10 \pm 0.93) \mathrm{mb}$ in $7 \mathrm{TeV}$ proton-proton collisions as obtained in Ref. [61] by extrapolating measurements reported in Ref. [62] using PYTHIA. This leads to enormous, and still growing, data sets of beauty and charm hadrons, with tens of millions of clean signal events. Such high-statistics data samples constitute a huge opportunity for high-precision flavor physics, but they also challenge the theoretical tools we have to analyze these data sets, including Dalitz plot and partial-wave analyses.

\subsection{Current analysis techniques}

\subsubsection{Diffraction and diffractive dissociation}

These processes - see Fig. 4 (left) - refer to production of resonances from dissociation of either the beam or the target. In a high-energy collisions, these can be kinematically separated due to a large rapidity gap. The following discussion focuses on meson production from dissociation of a high-momentum meson (pion) beam in the hadronic or Coulomb field of the target nucleus. The methodology equally well applies to the target fragmentation region.
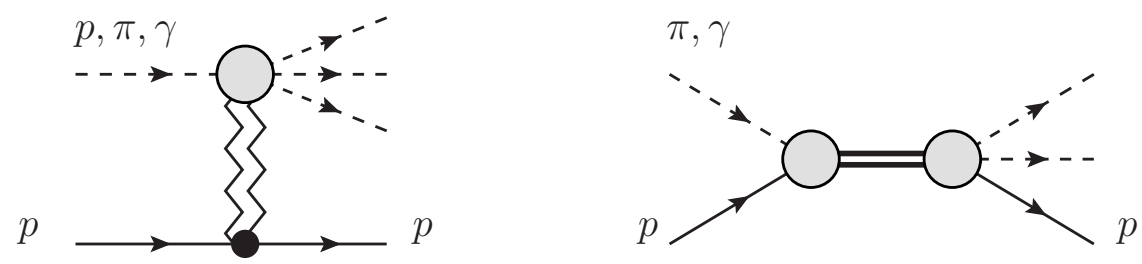

Fig. 4. Schematic representation of diffractive dissociation (left) and direct production (right).

The analysis typically starts with the selection of events corresponding to a given final state. Exclusive events are selected by imposing energy conservation between incoming and outgoing particles, and transverse momentum balance between incoming and outgoing particles including the recoil particle.

To disentangle the resonances contributing to a given final state, a partialwave analysis (PWA) is performed, which involves certain model assumptions. At high $\sqrt{s}$, the reaction can be assumed to proceed via $t$-channel exchange between the target and the projectile, which excites the projectile to a state $X$ and leaves the target intact. The state $X$ then decays into a multi- 
particle final state without further final-state interaction. This sequence is described by the phenomenological approach of the isobar model [63]. In this model, the production and the decay of a state $X$ with quantum numbers $\chi=I^{G}\left(J^{P C}\right) M$ factorize into a production amplitude $T_{\chi r}\left(t^{\prime}, s, m\right)$ and a decay amplitude $A_{\chi \zeta}(\tau, m)$, where $r$ summarizes the quantum numbers (e.g. helicities) of the projectile, the target, and the recoil, $t^{\prime}$ and $s$ are the reduced 4-momentum transfer squared and squared center-of-mass energy, respectively, $m$ is the invariant mass of the intermediate state $X$, and $\tau$ denotes the set of kinematic variables describing the decay of $X$ into a particular decay channel denoted by $\zeta$. The propagation of the intermediate state $X$ is described by a a propagator-like amplitude $K_{\chi}\left(t^{\prime}, s, m\right)$, which carries a phase and depends on $m$. The amplitude for observation of a given final state is then written as

$$
\mathcal{A}_{r}\left(t^{\prime}, s, m\right)=\sum_{\chi \zeta} T_{\chi r}\left(t^{\prime}, s, m\right) K_{\chi}\left(t^{\prime}, s, m\right) A_{\chi \zeta}(\tau, m),
$$

where the sum runs over all possible partial waves $T_{\chi r} A_{\chi \zeta}$, i.e. quantum numbers of intermediate states $X$ and decay channels $\zeta$ which lead to a given final state observed in the experiment.

Usually, the analysis is performed in a two-step approach. In the first step, the data are partitioned in small bins of $m$ and $t^{\prime}$, such that the production amplitudes can be assumed to be constant within each bin. For a fixed beam energy, i.e. $s$ fixed, and $s \gg t^{\prime}$, the propagator $K$ can be assumed to be approximately constant in a small mass bin and will hence be absorbed into the production amplitude. The amplitude for fixed $m$ and $t^{\prime}$ is then written as

$$
\mathcal{A}_{r}=\sum_{\chi \zeta} T_{\chi r} A_{\chi \zeta}(\tau)
$$

The decay amplitudes $A_{\chi \zeta}(\tau)$ can be calculated using the isobar model, in which the decay of $X$ is described as a series of sequential two-body decays into intermediate resonances (isobars), which eventually decay into the final state observed in the experiment. The sequence of two-body decays is calculated using a suitable spin formalism [64], taking into account isospin and Bose symmetry. An important feature of the two-body amplitudes is the factorization into an angular part, described e.g. by Wigner- $D$-functions, and a dynamical part. The dynamical part contains the respective ClebschGordan coefficients for a given two-body decay, a dynamical function parameterizing the dependence of the amplitude on the mass of the decaying state ("isobar parameterization"), and the partial-wave decay amplitude (sometimes also called "isobar factor") which represents the overlap of the wave function of the mother particle with the two-body wave function of the daughter particles. In the analysis framework used, these amplitudes are assumed to be independent of the two-body kinematics, and are thus absorbed 
into the respective production amplitudes, which then depend not only on the quantum numbers $\chi$ of $X$, but also on the specific decay channel $\zeta$, summarized as $\xi \equiv \chi \zeta$ in $T_{\xi r}^{2}$.

Complications to the isobar model arising from unitarity constraints are difficult to treat theoretically and are usually neglected with the argument that normally not all possible decay modes of the intermediate state $X$ are fit simultaneously, but only a small subset or even a single final state is considered. Unitarity in the two-body subsystems is also an issue if more than one (narrow) isobar is included for a given state $X$ because then the partialwave decay amplitudes may no longer be considered constant, but will depend on the two-body kinematics. An analysis of the reaction $\pi N \rightarrow \pi \pi N$ incorporating unitarity in the two-body isobar channels using dispersion relations [65] has come to the conclusion that the results are compatible with an analysis ignoring the constraints [66]. With the much larger data sets available today, this issue is certainly worth being revisited. Current work which focuses on implementing unitarity constraints in the isobar model are summarized in Sec. 3.

The isobars are typically parameterized using relativistic Breit-Wigner functions with mass-dependent widths (if branching ratios are known) or a Flatté ansatz. It is well known that this ansatz is not justified for the $\pi \pi$ scalar-isoscalar partial wave. Different authors provide different parameterizations for this important amplitude, see, e.g., Ref. [67, 68]. A lot of progress has been achieved towards a full theoretical control of this channel in recent years, $c f$. Sec. 3 .

A new method to determine the isobar dynamics directly from the data, developed in the framework of the COMPASS experiment, gives very promising results, but is at the moment limited to few isobars only due to a drastic increase of fit parameters.

The observed multi-differential angular distribution is written as the coherent sum over all partial-wave amplitudes leading to the same final state

$$
\frac{d \sigma}{d \tau} \propto \sum_{r}\left|\sum_{\xi} T_{\xi r} A_{\xi}(\tau)\right|^{2} \equiv I
$$

This general form also includes a sum over $r$, which allows for possible sources of incoherence in the production process, e.g. due to unobserved helicities of incoming particle, target or recoil, but also due to experimental effects such as finite resolutions. Defining the elements of the spin-density

${ }^{2}$ Note that the amplitudes $T_{\xi r}$ and $A_{\xi}$ are now different from the ones in Eq. (2), because the isobar factors have been moved from the decay amplitudes to the production amplitudes. To avoid unnecessary clutter, we use the same symbol for both amplitudes. 
matrix $\rho$ as

$$
\rho_{\xi \xi^{\prime}}=\sum_{r} T_{\xi r} T_{\xi^{\prime} r}^{*},
$$

the angular distribution Eq. (3) can be also written as

$$
I=\sum_{\xi \xi^{\prime}} \rho_{\xi \xi^{\prime}} A_{\xi}(\tau) A_{\xi^{\prime}}^{*}(\tau) .
$$

Based on the observed angular correlations of the final-state particles, the intensity in each bin is thus decomposed into partial waves with definite spin and parity, without any prior assumptions on the shape of the amplitude as a function of $m$ or $t^{\prime 3}$.

The analysis is commonly performed in a reference frame, in which the $y$ axis is normal to the production plane, and the $z$ axis, i.e. the quantization axis, is chosen along some preferred direction in the production plane, e.g. the direction of the beam or the recoil particle. In such a system, parity conservation in the scattering process is conveniently taken into account by using a basis of states with a definite symmetry under a reflection through the production plane, given by the reflectivity $\epsilon$, and defined as [69]

$$
|J M ; \epsilon\rangle=c(M)\left[|J M\rangle-\epsilon P(-1)^{J-M}|J-M\rangle\right]
$$

with $\epsilon= \pm 1$ for bosons (mesons), $c(M>0)=1 / \sqrt{2}, c(M=0)=1 / 2$, and $M$ the modulus of the spin projection onto a given axis. The reflectivity is defined such that, for beams with pseudoscalar particles, it corresponds to the naturality of the Regge trajectory exchanged in the scattering process. Parity conservation implies that states with different reflectivity do not interfere, and the intensity can then be written as

$$
I=\sum_{\epsilon} \sum_{\xi \xi^{\prime}} \rho_{\xi \xi^{\prime}}^{\epsilon} A_{\xi}^{\epsilon}(\tau) A_{\xi^{\prime}}^{\epsilon *}(\tau) .
$$

In principle, an infinite number of waves is needed in the partial-wave expansion of the cross section (7). A limited amount of data, however, requires a truncation of the series and hence a possibly biased selection of waves that are included in the fit. Larger data sets help to reduce the model bias because more waves can be included in the fit. A model-independent algorithm for the selection of the wave set, based on genetic evolution, was introduced in [70]. Also this algorithm, however, requires tuning of parameters and may hence contain some residual bias, but it is definitely superior

\footnotetext{
${ }^{3}$ If the data are not sufficient to make fine bins in both $m$ and $t^{\prime}$, the dependence of a given partial wave on $t^{\prime}$ is taken into account by specific functions $f_{\xi}\left(t^{\prime}\right)$.
} 
to the standard approach of selecting waves "by hand". The fit normally contains also a background wave, characterized by a uniform distribution in $n$-body phase space, which is added incoherently to the other waves. The need for the inclusion of those background terms is a clear indication that the amplitudes used are incomplete and further theoretical progress is necessary - some possible routes for future developments are outlined in Sec. 3.

In the first step of the analysis, an event-based extended log-likelihood fit of the probability density in the full final-state phase space is usually performed to determine the complex production amplitudes $T_{\xi r}$ for each bin of the final-state invariant mass $m$ and of the 4-momentum transfer $t^{\prime}$. The elements of the spin-density matrix are then calculated as $\rho_{\xi \xi^{\prime}}^{\epsilon}=\sum_{r} T_{\xi r}^{\epsilon} T_{\xi^{\prime} r}^{\epsilon *}$. The diagonal elements of the spin-density matrix are the intensities of the corresponding waves, while the off-diagonal elements determine the phase differences between two waves. The fit also takes into account the experimental acceptance of the spectrometer, calculated from a phase-space Monte Carlo simulation of the apparatus.

The result of the first step of the PWA is an independent spin-density matrix for each $m$ and $t^{\prime}$ bin, containing all waves used in this particular bin. Apart from reasons of model complexity and computing resources, the splitting in two steps has the advantage that no dependence of the amplitude on the mass $m$ is introduced in the first step. Therefore, apart from the assumed factorization into production and decay amplitudes, no assumptions about the resonances in the analyzed $n$-body system enter the analysis at this point, so that model dependence is kept at a minimum.

In the second step, a model is applied in a $\chi^{2}$ fit to describe the mass and $t^{\prime}$-dependence of these matrices, where for computational reasons only a few waves are considered. For each wave, the model includes resonant contributions, usually parameterized in terms of relativistic Breit-Wigner functions with dynamic widths and parameters independent of $t^{\prime}$, and nonresonant contributions added coherently, in most cases parameterized by empirical functions.

\subsubsection{Direct-channel production}

This refers to a situation when the center-of-mass energy of colliding particles coincides with a mass of nearby resonances, see Fig. 4 (right). Resonance decay is analyzed following the same ideas of isobar factorization discussed above. Direct-channel baryon excitations decaying to at most two pseudoscalar mesons and a baryon have often been analyzed by fitting model amplitudes to the partially integrated differential cross section and polarization observables. With new, high-statistics data now becoming available, an event-by-event analysis, similar to the one described above, ought to be performed. 
Most analyses have, so far, focused on decay channels containing a single meson and a baryon. Model amplitudes are often based on effective Lagrangians which incorporate low-energy, e.g. chiral and unitarity constraints. Two-meson production, especially $\pi \pi$ production, has the largest cross section for photoproduction from energies around the second resonance region and higher and, since it couples strongly to many baryon resonances, it is an important channel that complements the information obtained in single pseudoscalar meson photoproduction.

There are two main approaches to analyze data on pion or photo-induced production of single mesons. The first approach is the so-called energyindependent approach or single-energy solution. Here, data on differential cross sections and polarization observables are analyzed in fixed bins of energy (and ideally in fixed bins of solid angle) to extract the scattering or production amplitudes. When fixed bins in energy and solid angle are chosen, the so-called CGLN amplitudes emerge. For the definition of the CGLN amplitudes and their partial wave decompositions for pion-nucleon scattering [71] and photoproduction of a pseudoscalar [45], we refer the interested readers to the original publications. The model-independent determination of these amplitudes is the problem of the so-called complete experiment that has attracted much attention lately [72-74], including a dedicated workshop [75]. See Ref. [76] for a summary.

The angular dependence of the CGNL amplitudes yields in principle the photoproduction multipoles. The main problem is an undetermined overall phase of the multipoles, that is different not only for different energies but also for different angles. To disentangle multipoles, usually a truncation in multipoles is performed, or high partial waves from an existing analysis are held fixed, or a penalty function is used to guide the single-energy multipole extraction by an energy-dependent solution. Practically, the precision of existing data poses the major problem in the extraction of amplitudes. Single-energy solutions have been extracted for many years [77-79].

For pseudoscalar meson scattering, theoretically, only three observables need to be measured with good precision to reconstruct the scattering amplitudes up to one common phase. For the reaction $\pi^{-} p \rightarrow K^{0} \Lambda$, spin rotation parameters have been determined even though with limited solid-angle coverage. A Bonn-Gatchina fit to the data provided a set of single-energy solutions (6 per energy point) [80]. The solution closest to the energy-dependent solution was chosen as the physical one. This solution was smooth and very close to the energy-dependent solution. The second best solution showed larger fluctuations in all partial waves.

The situation is much more complicated in the case of photoproduction amplitudes. Here, at least eight observables are needed for an unambiguous extraction of the amplitudes. The single-energy approach is particularly suited for the analysis of reactions with kaon-hyperon final states, where the 
recoil polarization of the final baryon can be measured. Another possibility is the analysis of data on meson production taken at relatively low energies where the number of contributing multipoles is restricted and only few observables are needed to construct the amplitudes. In [81], this method was exploited to determine the helicity amplitudes of the $N(1520) 3 / 2^{-}$resonance from an energy-independent analysis of $S$-, $P$-, and $D$-waves in $\gamma p \rightarrow p \pi^{0}$.

The second approach is the so-called energy-dependent partial-wave analysis. Here, the angular and energy dependencies are analyzed simultaneously. A weak point of this approach is the large number of parameters and the large amount of computer time to obtain a solution. However, this approach offers also several advantages. First of all, global properties of the amplitudes - like unitarity and analyticity — can be imposed right from the beginning. The unitarity property is a powerful constraint when elastic and inelastic channels are analyzed jointly. As mentioned, a given resonance has one pole position, which is fixed in all reactions. Its couplings to the different channels define its contributions to the different pion- and photoinduced reactions. Energy-dependent coupled-channel analyses are thus the method of choice to search for weak resonance signals. Single-energy solutions, on the other hand, can be used to search for very narrow structures that tend to be missed by energy-dependent analyses [77].

At this point, it should be stressed that better data for pion-induced reactions are of urgent need. In coupled-channel analyses, these data determine the hadronic part of the amplitude and, consequently, also the photoand electroproduction amplitudes. However, many measurements of pioninduced reactions date back to twenty or more years ago. Often, systematic uncertainties are not reported, or are known to be underestimated. The need for hadronic beams has been recently discussed on a dedicated workshop [82].

Groups such as SAID, MAID, Jülich/Athens/GWU, EBAC (now Argonne-Osaka), Gießen, and Bonn-Gatchina have all made valuable contributions to this field, and anticipate being able to utilize further data as it becomes available.

In the following section, we detail some of the theoretical constraints that are being developed and that need to be implemented to verify the various assumptions of these analysis techniques.

\section{Amplitude analysis}

[C. Hanhart]

Hadron spectroscopy aims at the identification of hadron resonances and the determination of their properties. In the limit of a large number of colors, hadrons become bound states of constituent quarks. In reality, almost all of them are resonances that decay strongly to ground state hadrons 
- pions, kaons, etas, and nucleons. The heavier the resonance, the more multi-particle channels are allowed kinematically as final states. As a result, resonances become broad, overlap, and their identification gets increasingly difficult. The goal of the amplitude analyses outlined here is to pin down the spectrum in the so-called resonance region which typically corresponds to excitation energies not greater than $2-3 \mathrm{GeV}$.

The easiest and most commonly used parametrization for decays and scattering amplitudes is built from sums of Breit-Wigner functions (BWs) with energy-dependent widths, sometimes accompanied by (smoother) background terms. While this ansatz typically allows for a high-quality fit of many-body final states, it suffers from various problems. The poles of the BWs are, in general, not identical to the true poles of the $S$-matrix. As such, their parameters may differ between different reactions, which prevents a systematic, consistent study of many final states. Typically, BWs do not reproduce the analytical properties of reaction amplitudes. In addition, sums of BWs violate unitarity. For instance, in the case of $2 \rightarrow 2$ scattering, unitarity correlates the energy-dependent complex phases between the different BWs. In decays with only two strongly-interacting particles in the elastic regime, Watson's theorem imposes the equality between scattering and production phases. Therefore, sums of BWs may only be a valid approximation when considered far from kinematic thresholds and only for poles close to the real axis that are far from each other, that is for narrow and isolated resonances.

In this section, we outline theoretical aspects that need to be considered to arrive at parameterizations of amplitudes that try to minimize the effect of the above-mentioned problems. From the point of view of reaction theory, also known as $S$-matrix theory, resonances are poles of partial-wave scattering amplitudes in the unphysical domain of kinematical variables, energy, and/or angular momenta. Thus, their identification requires an analytic extension of the (multi-channel) amplitudes into the complex plane of the kinematic variables. $S$-matrix theory imposes severe constraints on the amplitudes allowed, such as unitarity, analyticity, as well as crossing symmetry. In addition, the amplitudes have to be consistent with the assumed discrete symmetries of the underlying theory. Depending on the kinematical regime of an experiment, different aspects of this list may become relevant. For example, low-energy scattering is dominated by a few elastic partial waves, which may be constrained by unitarity, analyticity, and in some special cases crossing symmetry ( $c f$. Sec. 3.1 on dispersion theory). To control subleading singularities, or if there is no sufficient information about particle scattering available to employ dispersion theory, in addition to the general principles, it is sometimes necessary to impose further properties on the reaction dynamics, e.g. from long-ranged meson exchanges whose strength may be constrained from data (e.g. the strength of the pion exchange in $\pi \rho \rightarrow \rho \pi$ 
is given by the width of the $\rho$ meson) or by chiral symmetry (cf. Sec. 3.2 on dynamical coupled-channel methods and related approaches). On the other hand, a detailed understanding of resonance production with high-energy beams may require knowledge of singularities in the complex angular momentum plane - Reggeons ( $c f$. Sec. 3.3 on duality and finite-energy sum rules).

In general, amplitude analysis can be considered as a three-step process. In step one, theoretical amplitudes are proposed and constrained by fitting the experimental data. In step two, these amplitudes are tested against various constraints that are used to minimize the amount of unresolved ambiguities in the amplitude determination. Finally, in step three, the amplitudes are extrapolated (analytically continued) to the unphysical kinematical region of energy and angular momentum to determine properties of resonances.

With the advent of new high-statistics experiments, combined with the development of theoretical tools, the widely used isobar model can now be replaced by model-independent analyses. Connecting the emerging lattice results with the parameters extracted using the analysis techniques mentioned above will provide a direct contact between experimental data and QCD.

\subsection{Dispersive methods}

[C. Hanhart, M. Hoferichter, B. Kubis, J.R. Peláez]

In this section, we will discuss several examples where dispersion relations (DRs) have been applied with the aim of obtaining precision parameterizations of amplitudes at low energies and performing their analytic continuation. Another important aspect that concerns the connection of low-energy physics and the high-energy region within dispersion theory will be touched upon in Sec. 3.3.

A resonance is uniquely characterized by its pole and residues, the position of the pole being universal, its residues depending on the decay channel in question. The challenge in the precision determination of these parameters lies in the restriction that experiments are limited to real, physical values of the center-of-mass energy $s$. In principle, DRs provide a rigorous way of analytically continuing amplitudes from the physical regime into the complex plane, and thus of unambiguously extracting the pole parameters of the resonance. Only when a resonance is well isolated from others and is also far from thresholds, one can use simple expressions like Breit-Wigner amplitudes that provide, in a limited region, a very good approximation to the result one would obtain from dispersion theory. Mathematically, these are cases where the distance of the resonance pole to the real axis is smaller than its distance to any other singularity, or where there is just one threshold cut nearby. Resonances corresponding to such a situation have been thoroughly studied and their properties are well established. Nowadays, we are 
trying to understand the complicated part of the spectrum, where this ideal situation often does not occur and resonances are wide, with poles relatively deep in the complex plane. Effects of overlapping resonances and proximity to more than one threshold due to many possible decay channels require more elaborate techniques.

For a general introduction to dispersive techniques, we refer to Refs. [83-85]. Briefly, in terms of physics, DRs are a consequence of causality, which mathematically allows us to analytically extend the amplitudes into the complex plane, and then use Cauchy's theorem to relate the amplitude at any value of the complex plane to an integral over the (imaginary part of the) amplitude evaluated on the real axis, where data are available. Such a relation can be used in several ways. On the physical real axis, it implies that the amplitude has to satisfy certain integral constraints. Thus, one can check the consistency, within uncertainties, of the data at a given energy against the data that exist in other regions. Additionally, DRs may be imposed as constraints, by forcing the amplitude to satisfy the DR while fitting the data. Finally, certain sets of coupled DRs are so strongly constrained (see the discussion of Roy equations below) that they can actually be solved as a boundary problem in a limited (typically low-)energy range, given a specific high-energy input and depending on a well-defined number of parameters (subtraction constants) [86-89].

Especially, one can even use a DR to obtain values for the amplitude at energies where data do not exist, using existing data in other regions. Once one has an amplitude that satisfies the DR and describes the data well, it is possible to extend the integral representation to obtain a unique analytic continuation into the complex plane (or at least to a particular region of the complex plane where the validity of the DR can be rigorously established). For partial-wave amplitudes, one can thus study the complexenergy plane and look for poles and their residues, which provide the rigorous and observable-independent definition for the resonance mass, width, and couplings.

Prime examples for precision determinations of resonance pole positions by dispersive techniques concern the $\sigma$ or $f_{0}(500)[90,91]$ as well as the $\kappa$ or $K_{0}^{*}(800)$ resonance [92]. While both are still "simple" in the sense that they are overwhelmingly dominantly elastic resonances (in $\pi \pi$ and $\pi K$ scattering, respectively), their poles are non-trivial to determine since they lie far away from the real axis, with widths of about $550 \mathrm{MeV}$ in both cases. By convention, the width $\Gamma$ of a resonance is defined as $\Gamma=-2 \operatorname{Im} \sqrt{s_{\mathrm{p}}}$, where $s_{\mathrm{p}}$ denotes the complex pole position of the resonance. The (complex) range of validity of the corresponding DRs is restricted by the singularities of the so-called double-spectral region, as well as by the requirement of the partialwave projection to converge, and can be shown to still comprise the poles under investigation. One furthermore employs the consequence of unitarity 
that poles of the $S$-matrix on the second Riemann sheet correspond to zeros on the first sheet; the positions of the latter are determined in practice. As the partial waves in these cases are given by DRs using imaginary parts along the real axis only, with kernel functions known analytically, this procedure is then straightforward.

DRs have been extensively studied for various $2 \rightarrow 2$ reactions, with a few extensions to include more complicated final states [93]. Amplitudes for twobody reactions depend on the Mandelstam variables $s$ and $t$ (or $u$ ), which are related to center-of-mass energy and momentum transfer, respectively. Typically, DRs are formulated in terms of $s$, with the $t$-dependence either fixed or integrated over. The former are referred to as "fixed- $t$ DRs". Of special importance among these kinds of DRs is the case $t=0$ for elastic reactions, known as "forward DRs", since, due to the optical theorem, the imaginary part of the forward amplitude is proportional to the total cross section, and data on total cross sections are generically more abundant and of better quality than on amplitudes for arbitrary values of $s$ and $t$.

On the other hand, one can eliminate $t$ by projecting the amplitude onto partial waves, for which then a DR is written. The advantage of these partial-wave DRs is that their poles on the second Riemann sheet are easily identified as resonant states with the quantum numbers of the partial wave. Therefore, they are very interesting for spectroscopy. However, due to crossing symmetry, partial waves have a left-hand cut in the unphysical $s$ region, which also contributes to the DR. If the region of interest lies very far from this cut, it can be neglected or approximated, but when closer, or if one wants to reach a good level of precision, it becomes numerically relevant and has to be taken into account. Since the amplitude in the unphysical region may correspond to different processes arising from crossed channels in other kinematic regions and other partial waves, this complicates the construction of DRs substantially. Dealing rigorously with the left-hand cut usually involves an infinite set of coupled integral equations, known for $\pi \pi$ scattering as Roy equations [94], but other versions exist for $\pi K \rightarrow \pi K, \gamma \gamma \rightarrow \pi \pi$, and $\pi N \rightarrow \pi N$, under the generic name of Roy-Steiner equations [95, 96]. There is a considerable and relatively recent progress, as well as growing interest in obtaining rigorous dispersive descriptions of these processes [88, 89, 97-105], which play an essential role when describing final states of almost all other hadronic strongly-interacting reactions.

In all these variants of DRs, the integrals formally extend to infinity. In order to achieve convergence and also to suppress the high-energy contribution, one introduces so-called subtractions. In a subtracted version of a given DR, the integrand is weighted by additional factors of $1 /\left(s-s_{0}\right)$, where $s_{0}$ is referred to as the subtraction point, at the expense of introducing a priori undetermined parameters (subtraction constants). For a $2 \rightarrow 2$ scattering process, in general, two subtractions are required to ensure 
convergence [106, 107], but once- or even less-subtracted relations exist for certain amplitudes. Subtraction constants can be constrained by matching to effective field theories, lattice calculations, or simply fits to data. For the high-energy region, one typically makes use of Regge theory, which is known to describe data on, for instance, total cross sections up to very large energies well. Even if data are not very precise or non-existent, Regge theory allows for predictions for different processes by combining the results for well-established reactions by means of factorization. Regge predictions are less robust for the $t$-dependence of the amplitudes, although if only small $t$ are required, they provide a reasonable approximation. Simple and updated Regge parameterizations can be found in the Review of Particle Physics [1], except for meson-meson scattering for which we refer to [100, 108-110].

Since most hadronic observables involve pions, kaons, or light nuclei in the final state, at some stage their theoretical description requires input from elastic $\pi \pi, \pi K$, and $\pi N$ scattering via the so-called Fermi-Watson theorem [111, 112]. For processes with only two strongly-interacting finalstate particles, it fixes the phase of the whole amplitude to that of the hadron pair. A rigorous dispersive implementation of this theorem can be achieved via the Muskhelishvili-Omnès (MO) method [113, 114], where the amplitude is expressed in terms of an Omnès factor uniquely determined by the phase of the scattering process of the final state. This method is particularly well-suited for the study of meson form factors, not only of pions, kaons, but charmed $D$ mesons as well, see, for instance, [115-122] and references therein. In addition to the right-hand cut accounted for by the MO method, the description of production amplitudes involves a lefthand cut. It should be stressed that the structure of this left-hand cut is different from the left-hand cut of the pertinent scattering reaction.

Building upon MO techniques, one may obtain a consistent treatment of $\pi \pi$ rescattering for more complicated reactions as well, e.g. using KhuriTreiman techniques for three-particle decays [123]. If for a given decay the contribution from the left-hand cut is known to be suppressed, e.g. for $\eta, \eta^{\prime} \rightarrow \pi^{+} \pi^{-} \gamma$ [124], and can be expanded in a polynomial, this setup reduces to the original MO solution, while otherwise coupled integral equations need to be solved. These integral equations happen to be linear in the subtraction constants, so that the full solution can be reconstructed by a linear combination of basis functions that correspond to the choice of one subtraction constant set equal to 1 and the others put to zero. In this way, one obtains a description of the amplitude in terms of a few parameters which can be determined by comparison to experiment, see [125] for the example of $\gamma \pi \rightarrow \pi \pi$. For a real decay process, the solution of the integral equations is further complicated by the analytic properties of the amplitude, which require a careful choice of the integration contour in the complex plane. For an application of these methods to $\eta, \omega, \phi \rightarrow 3 \pi$ decays, see [126-130]. 
Watson's final-state theorem as well as the more general consequences thereof encoded in the use of MO and Khuri-Treiman techniques only apply in the region of elastic unitarity (or at least as long as inelastic effects are sufficiently small to be negligible). In principle, the MO method can be generalized to multiple coupled channels, provided the corresponding multichannel $T$-matrix is known. In practice, this has been implemented mainly for the case of the $\pi \pi$ isospin $I=0 S$-wave, where the inelasticity sets in sharply at the $\bar{K} K$ threshold, which, at the same time, almost coincides with the position of the $f_{0}(980)$ resonance. In this case, the additional input needed beyond the $\pi \pi$ scattering phase shift are modulus and phase of the $\pi \pi \rightarrow \bar{K} K$ transition. Applications have mainly concerned scalar form factors of different kinds [131-135]. For the $\pi K$ system, strangeness-changing scalar form factors have been studied, taking the coupling to $\eta K$ and $\eta^{\prime} K$ into account $[136,137]$. It needs to be said, though, that this method can be realistically applied mainly in contexts where inelasticities are dominated by one or two channels; compare also the suggestion to approximate the coupling to additional channels via resonances only [116]. The combination of the Khuri-Treiman method to treat three-body decays with inelastic channel coupling has not been undertaken to date.

For more complicated processes, a rigorous formulation of DRs soon becomes extremely demanding. In such a situation, one could try to use models that incorporate at least the most relevant analytic structure, impose further constraints in the form of sum rules, and make sure that the resonances claimed lie within the applicability of the approach. Some models, based on simplified DRs, as for instance the $N / D$ method or some unitarized models, can be very useful to obtain resonance poles and parameters in cases with coupled channels, at least in those channels where reliable data exist. By all means, one should refrain from making spectroscopic claims from simple models that fail to obey these constraints.

\subsection{Dynamical coupled channels, Chew-Mandelstam, K-matrix, and related approaches in baryon analyses}

\section{[M. Döring]}

The phenomenology in the analysis of excited baryons is complex. One complication arises from known strong inelasticities into multi-pion states, mostly $\pi \pi N$. For example, two pions with the $\rho(770)$ quantum numbers are known to be responsible for inelasticities at higher energies. The two pions and the nucleon can also be in relative $S$-wave, i.e. one can have the effective quantum numbers of a $\sigma N$ state. With the centrifugal barrier absent, this configuration leads to large inelasticities into the $\pi \pi N$ channel even at low energies, resulting in the unusual resonance shape of the (very light) Roper resonance $N(1440) 1 / 2^{+}$. In other words, from the standpoint of meson 
spectroscopy, one has maximal contamination from excited baryons, while from the standpoint of baryon analysis, two-body channels as $\pi N$ need to be supplemented with three-body states.

Furthermore, a two-particle subsystem of the $\pi \pi N$ system can also contain resonance singularities. As mentioned, the $\pi \pi$ subsystem can have the quantum numbers of a $\rho(770)$, coupling to the nucleon with a certain isospin, total spin, and total angular momentum. In general, more than one configuration is possible. Those singularities lead to branch points in the complex plane of the overall center-of-mass scattering energy. These non-analyticities are located on the same sheets as resonances and can lead to false resonance signals if not properly taken into account [138]. Last but not least, the inelasticities from channels formed by a stable baryon and a stable meson are important. The prime example is the strong coupling of the $\eta N$ channel to the $S_{11}$ partial wave, in particular the $N(1535) 1 / 2^{-}$.

The complex phenomenology of the baryon resonance region has, so far, hindered the implementation of the rigorous methods discussed in previous sections. Also, the search for new baryon resonances usually implies a multichannel fit to data of different reactions, to look for resonances that couple only weakly to the $\pi N$ channel. Recently, experimental activity has focused on photo- and electroproduction reactions, with a variety of final states such as $\pi N, \pi \pi N, \eta N, \pi \eta N, K \Lambda, K \Sigma$, and $\omega N$. As resonance pole positions are independent of the reaction studied, the simultaneous analysis of different final states facilitates the search for weak resonance signals.

Several analysis tools have been developed for the analysis of excited baryons, among them the so-called dynamical coupled-channel approaches, pursued in the ANL-Osaka (former EBAC) Collaboration, in the JülichAthens-GWU Collaboration, in the Dubna-Mainz-Taipeh (DMT) group, and others [139-142]; see Fig. 5 for recent results. The left-hand cuts are approximated perturbatively by $u$-channel baryon exchanges, while $s$-channel unitarity, driven by the right-hand cut, is respected exactly. The discussed $\pi \pi N$ three-body states are included such that two-body subsystems describe the corresponding phase shifts. Subthreshold non-analyticities such as the circular cut, short nucleon cut, and further left-hand cuts are present. Exchanges in $t$ - and $u$-channel are truncated to the lightest (excited) hadrons. These exchanges provide a background that connects different partial waves and limits the room for resonances. In the Jülich approach, the $t$-channel dynamics for the $\rho$ and $\sigma$ quantum numbers is provided by the use of dispersive techniques and a fit to $N \bar{N} \rightarrow \pi \pi$ data [143].

Another aspect of three-body dynamics is the consistent implementation of two-body decays. It has been shown [144] that unitarity in the three-body sense can be achieved by complementing three-body states with appropriate exchange processes. For example, in the three-pion system, a $\pi \rho(770)[\pi \pi]$ state requires appropriate pion exchanges to fulfill unitarity. That princi- 


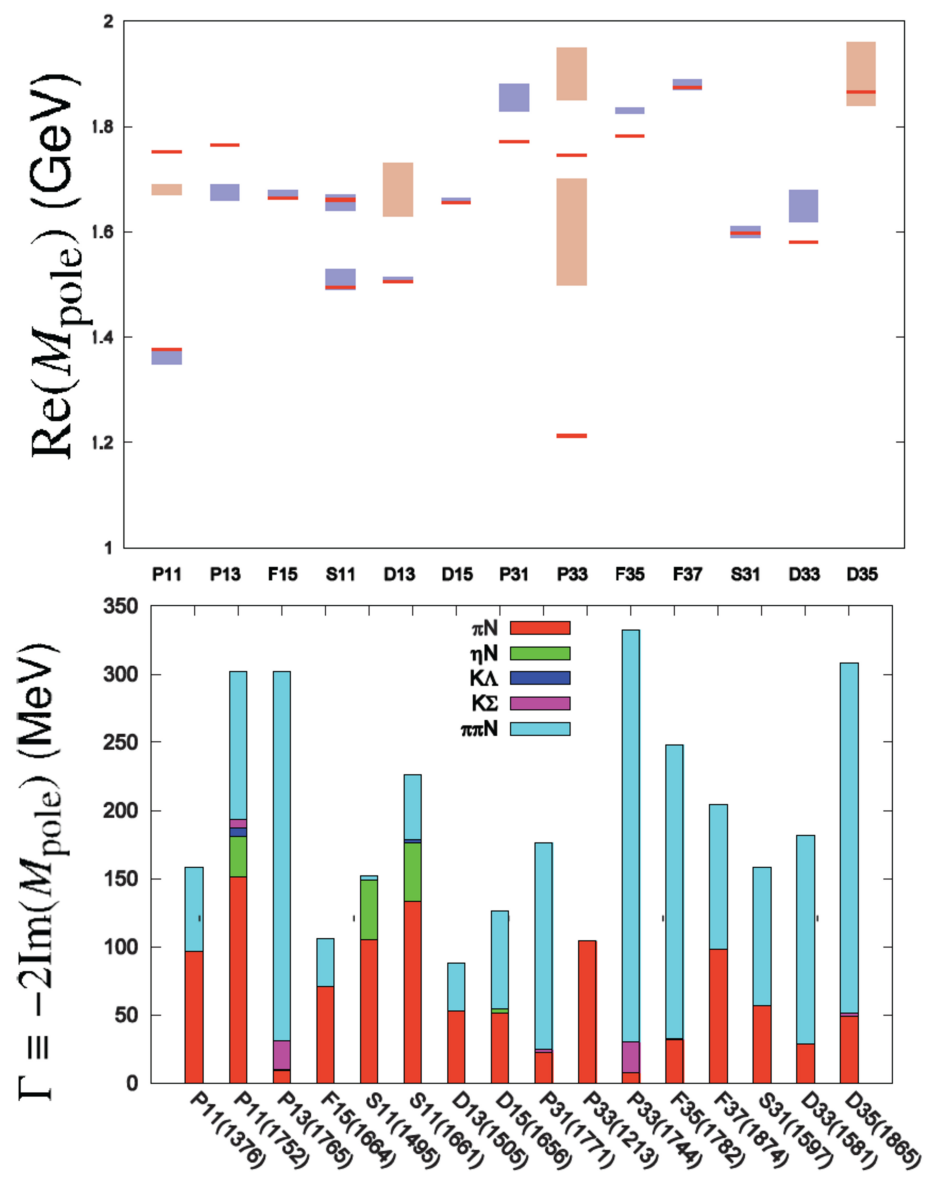

Fig. 5. Baryon spectrum: masses (left) and widths (right) from the ANL/Osaka approach [139].

ple has inspired the construction of dynamical coupled-channel approaches in baryon analysis as well $[139,140]$. In meson analysis, three-body unitarity has been explored in [145], using effective Lagrangians and isobars, that fulfill two-body unitarity and fit the corresponding phase shifts. If one restricts the rescattering series to the first term, one recovers an amplitude closely related to the traditional isobar picture that may or may not be unitary in the two-body sense, but is never unitary in the three-body sense. Summing up, the consistently constructed interaction beyond the leading term, including rearrangement graphs, restores unitarity in the three-body sense. See also [146], where three-body unitarity based on point-like interactions is considered. Coming back to the analysis of excited baryons, three-body unitarity is sometimes not manifestly included but effectively 
approximated by free phases as in the $D$-vector approach by the BonnGatchina group. Similarly, in the MAID analysis, unitarity is approximated by complex phases [79].

In dynamical coupled-channel approaches, usually a scattering equation with off-shell dependence of the driving interaction is solved. If the interaction is factorized on-shell, the integral equation reduces to a matrix equation in coupled channels. Real, dispersive parts of the intermediate propagating states can be maintained. Such contributions are relevant for the reliable analytic continuation to search for resonance poles and residues.

Analyses of this type are pursued by the GWU/INS (SAID) approach in the Chew-Mandelstam formulation [77, 78], by the Bonn-Gatchina group in the $N / D$ formulation [147, 148], and by the Kent State [149] and the Zagreb [150] groups in the Carnegie-Mellon-Berkeley (CMB) formulation. The Gießen group uses a $K$-matrix formalism [151, 152], while the MAID approach employs a unitary isobar approach in which the final-state interaction is taken from the SAID approach [79]. Dispersive approaches and unitary isobar analyses on meson electroproduction have been performed by the JLab group [153]. Two-pion electroproduction is analyzed at JLab as well [154].

In the GWU/INS (SAID) approach, the interaction is parameterized without the need of explicit resonance propagators [78]. Resonance poles are generated only if required by data, which makes this approach particularly model-independent for baryon spectroscopy.

The Bonn-Gatchina approach, formulated with covariant amplitudes [155], performs combined analyses of all known data on single- and doublemeson photo- and pion-induced reactions (see, e.g., Ref. [156]); four new states [147] were reported recently. See Fig. 6 for an overview. In the BonnGatchina approach, fits to reactions with two-body final states are carried out by minimization of $\chi^{2}$ functions, while the multi-body final states are analyzed in an event-by-event maximum likelihood method which fully takes into account all correlations in the multi-dimensional phase space.

The Gießen group has recently included the analysis of $\pi \pi N$ data in form of invariant mass projections [157], similar to the previous work of the EBAC [158] group, while the original Kent State [159] analysis uses events directly.

In the search for excited baryons, considerable progress has been made in the analysis of the corresponding data. In particular, recent data with unprecedented accuracy from ELSA, JLab, MAMI, and other facilities have improved the precision determination of resonance parameters. Still, no consensus has been reached on the resonance content, in particular for broad resonances or those that couple only weakly to the analyzed channels, $c f$. Fig. 6. It is expected that additional constraints from crossed channels and analyticity in complex angular momenta will help improve the reliability of 
resonance extraction and determination of the spectrum. This is particularly relevant for the data in forward direction and at higher energies. Here, a matching of Regge amplitudes and unitary methods is a promising way to provide the correct asymptotic behavior.

J. Beringer et al. (Particle Data Group), Phys. Rev. D86, 010001 (2012).

\begin{tabular}{|c|c|c|c|c|c|c|c|c|}
\hline Resonance & Rating & $N_{\text {pp }}$ & Resonance & Rating & $N_{\text {pp }}$ & Resonance & Rating & $N_{\text {pp }}$ \\
\hline $\mathrm{N}(1440) 1 / 2^{+}$ & $x \times x x$ & 13 & $N(1520) 3 / 2^{-}$ & $x x x x$ & 17 & $N(1535) 1 / 2^{-}$ & $x \times x x$ & 15 \\
\hline$N(1650) 1 / 2^{-}$ & $\pi x \times x$ & 18 & $N(1675) 5 / 2^{-}$ & $x \times x \times$ & 14 & $N(1680) 5 / 2^{+}$ & $x \times x \times$ & 17 \\
\hline$N(1685)$ & x & & $N(1700) 3 / 2^{-}$ & $x \times x$ & 15 & $N(1710) 1 / 2^{+}$ & $x \times x$ & 14 \\
\hline $\mathrm{N}(1720) 3 / 2^{+}$ & $x \times x \times$ & 17 & $N(1860) 5 / 2^{+}$ & $\mathrm{xx}$ & 9 & $N(1875) 3 / 2^{-}$ & $x \mathbf{x x}$ & 16 \\
\hline $\mathrm{N}(1880) 1 / 2^{+}$ & $x x$ & 20 & $N(1895) 1 / 2^{-}$ & $x x$ & 17 & $N(1900) 3 / 2^{+}$ & $x \times x$ & 18 \\
\hline $\mathrm{N}(1990) 7 / 2^{+}$ & $\pi x$ & 9 & $N(2000) 5 / 2^{+}$ & $x x$ & 11 & $N(2040) 3 / 2^{+}$ & $x$ & \\
\hline$N(2060) 5 / 2^{-}$ & $x x$ & 13 & $N(2100) 1 / 2^{+}$ & x & & $N(2150) 3 / 2^{-}$ & $x$ & 11 \\
\hline $\mathrm{N}(2190) 7 / 2^{-}$ & $x \times x \times$ & 11 & $\mathrm{~N}(2220) 7 / 2^{-}$ & $x \times x$ & 7 & $N(2250) 9 / 2^{-}$ & $x \times x \times$ & \\
\hline$N(2600) 11 / 2^{-}$ & $x \times x$ & & $\mathrm{~N}(2700) 13 / 2^{+}$ & $x$ & & & & \\
\hline$\Delta(1232)$ & $x \times x \times$ & 8 & $\Delta(1600) 3 / 2^{+}$ & $x \times x$ & 12 & $\Delta(1620) 1 / 2^{-}$ & $\mathbf{x x \mathbf { x } x}$ & 10 \\
\hline$\Delta(1700) 3 / 2^{-}$ & $x \times x \times$ & 11 & $\Delta(1750) 1 / 2^{+}$ & × & & $\Delta(1900) 1 / 2^{-}$ & $\mathrm{xx}$ & 13 \\
\hline$\Delta(1905) 5 / 2^{+}$ & $\pi x \times x$ & 11 & $\Delta(1910) 1 / 2^{+}$ & $x \times x$ & 13 & $\Delta(1920) 3 / 2^{+}$ & $x \times x$ & 21 \\
\hline$\Delta(1930) 5 / 2^{-}$ & $x \times x$ & & $\Delta(1940) 3 / 2^{-}$ & $\times$ & 5 & $\Delta(1950) 7 / 2^{+}$ & $\mathbf{x x x}$ & 13 \\
\hline$\Delta(2000) 5 / 2^{+}$ & $x x$ & & $\Delta(2150) 1 / 2^{-}$ & × & & $\Delta(2200) 7 / 2^{-}$ & $\times$ & \\
\hline$\Delta(2300) 9 / 2^{+}$ & $\pi x$ & & $\Delta(2350) 3 / 2^{-}$ & * & & $\Delta(2390) 7 / 2^{+}$ & $x$ & \\
\hline$\Delta(2420) 11 / 2^{+}$ & $x \times x \times$ & & $\Delta(2400) 9 / 2^{-}$ & $x x x$ & & $\Delta(2750) 13 / 2^{-}$ & 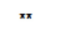 & \\
\hline$\Delta(2950) 15 / 2^{+}$ & $x x$ & & & & & & & \\
\hline
\end{tabular}

E.g.: V. Kuznetsov et al., Phys. Lett. B 647, 23 (2007); V. Kuznetsov et al., Phys. Rev. C 83, 022201 (2011); I. Jaegle et al., Eur. Phys. J. A 47, 89 (2011). M. Ablikim et al. [BES Collaboration], Phys. Rev. D 80, 052004 (2009).

A. V. Anisovich, R. Beck, E. Klempt, V. A. Nikonov, A. V. Sarantsev and U. Thoma, Eur. Phys. J. A 48, 15 (2012);

$N_{\text {pp }}$ particle properties were determined; 400 in total. Be cautious, there are ambiguities !

Promoted to three-star resonance

Fig. 6. Baryon spectrum from the Particle Data Group with certain new states from the Bonn-Gatchina analysis [148] and others.

Another direction in which systematic uncertainties underlying these phenomenological analyses can be quantified is to test whether the amplitudes satisfy $S$-matrix analyticity as expressed by finite-energy sum rules (FESR). Forward dispersion and other relations are included in the GWU/ INS (SAID) approach [160].

Despite the rather involved phenomenology and the conceptual differences of the discussed baryon analysis tools, there are indications that results become eventually consistent among different groups [161], and that the long-sought determination of the baryon spectrum gets within reach. The expected additional double polarization data from leading photoproduction experiments should provide a more consistent picture of baryon states up to masses of about $2.2 \mathrm{GeV}$. 


\subsection{Duality and finite-energy sum rules}

[G. Fox, V. Mathieu, A. Szczepaniak]

In the preceding sections, we focused on those $S$-matrix properties that are most important at low energies. Specifically, we discussed how, at the level of partial waves, to employ analyticity in order to implement unitarity and use effective Lagrangians to implement various symmetries.

The number of relevant partial waves grows with increasing channel energy and in reactions that, at least in some channels, involve large Mandelstam invariants a large (infinite) number of partial waves will contribute. As shown by Regge, high-energy behavior in a direct channel is dual to resonances in overlapping crossed channels. The crossed-channel resonance contributions can be expressed in terms of Regge poles and cuts, often referred to as Reggeons. The locations and properties of Reggeons are constrained by analyticity of partial waves continued to the complex angular momentum plane.

Schematically, as a function of channel energy variable, $s$, reaction amplitudes can be separated into a contribution from the low-energy region, where the $s$-dependence can be parameterized with a finite number of partial waves, and the high-energy region, where the amplitude is determined through Reggeons. The low-energy partial waves contain information about directly produced resonances, whereas Reggeons know about resonances in crossed channels. To eliminate possible double counting, the low-energy partial waves need to be removed from the high-energy Reggeon contributions. Analyticity is then used to constrain the two regions. That is, with all other kinematical variables fixed, the amplitude is an analytical function of channel energy with singularities originating from bound states and opening of physical thresholds. This enables one to write dispersion relations that connect the low-energy partial waves with the high-energy Reggeons. The energy dependence of such DRs is often converted into a set of moments and used as sum rules, also known as finite-energy sums rules (FESR) [162, 163] that relate parameters of resonances in direct and crossed channels. The classic application of FESR was in charge exchange $\pi N$ scattering [164, 165], and used to establish a relation between the leading, $\rho$ meson, and $\pi N$ resonances.

The observation that the low-energy contribution to FESR when saturated by resonances reproduces the contribution from leading Reggeons at high energy led to the concept of duality [166, 167]. According to this hypothesis, directly produced resonances in low partial waves are dual to Reggeons, and residual, non-resonant backgrounds are dual to the Pomeron. This hypothesis is consistent with what is expected in the limit of a large number of colors and the valence quark model. It is, therefore, worth noting that the existence of various exotic resonances that cannot be accommo- 
dated within the quark model would also lead to violations of this simple two-component duality. FESR studies can thus provide additional arguments in favor of or against the existence of new resonances. As an example, let us consider $K^{+} p$ elastic scattering. Directly produced resonances manifest themselves in the large imaginary part of the amplitude. The $K^{+} p$ direct channel has strangeness +1 and the absence of flavor exotic baryon resonances implies relations between crossed-channel Reggeons that enforce the vanishing of the Reggeon contributions to the imaginary part of the amplitude. These are known as exchange degeneracies (EXD), and in the case of $K^{+} p$, involve the $\rho$ and $a_{2}$ Regge trajectories. Similarly, destructive interference between the $\rho$ and the $\sigma$ (nowadays called $f_{0}(500)$ ) resonances in a direct channel is consistent with the absence of isospin 2 resonances in $\pi \pi$ scattering in a crossed channel [168]. The effect can be observed, for example, in the $3 \pi$ Dalitz distribution obtained from $\pi^{-}$diffractive dissociation, as illustrated in Fig. 7 [169].

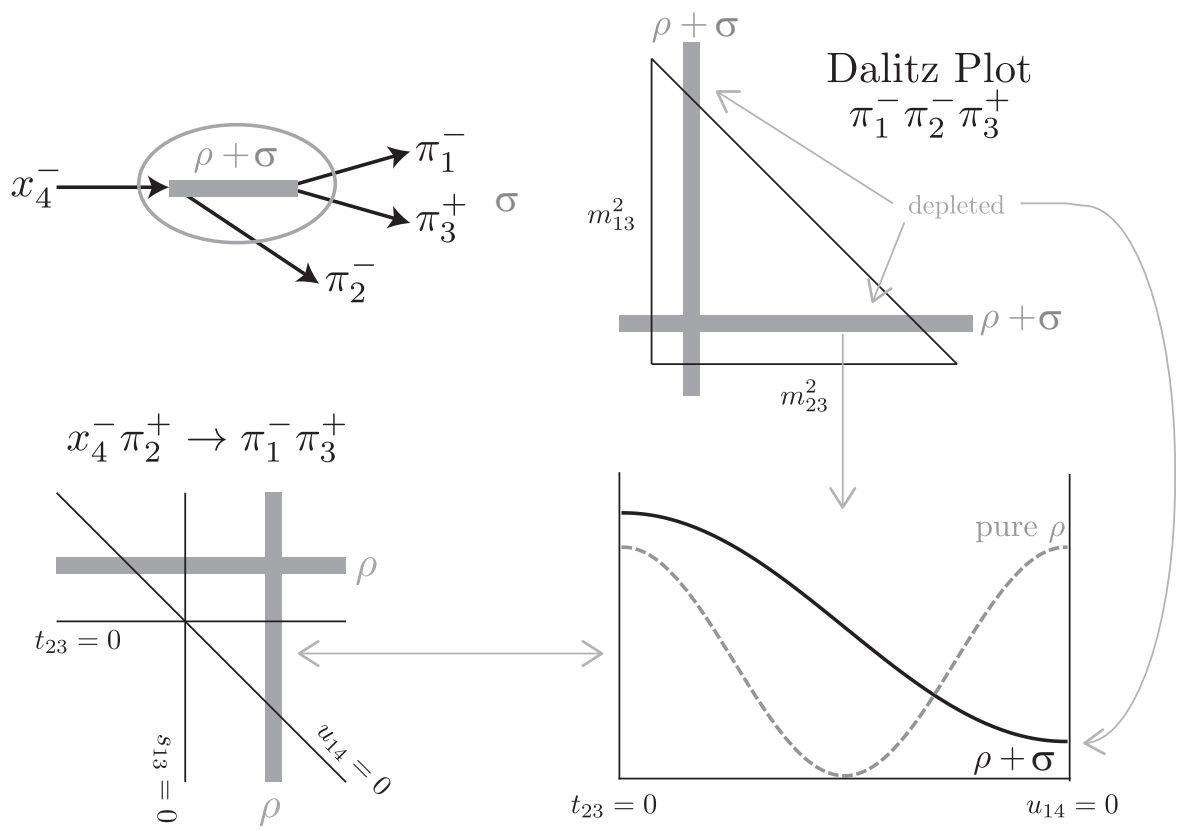

Fig. 7. The $\rho$ and the $\sigma$ must interfere coherently to suppress double charge exchange in the $\pi_{4}^{-} \pi_{3}^{-} \rightarrow \pi_{1}^{-} \pi_{2}^{-}$channel. The $\sigma$ refers to the lowest isospin-0 $\pi \pi$ resonance.

FESR can also be used to distinguish what is background and what is a $q \bar{q}$ resonance. As illustrated in Fig. 8, the final-state interactions generating resonances in the low-spin partial waves in the 23 channel are dual to the $\rho^{0}$ and $\sigma$ in the 13 channel. 


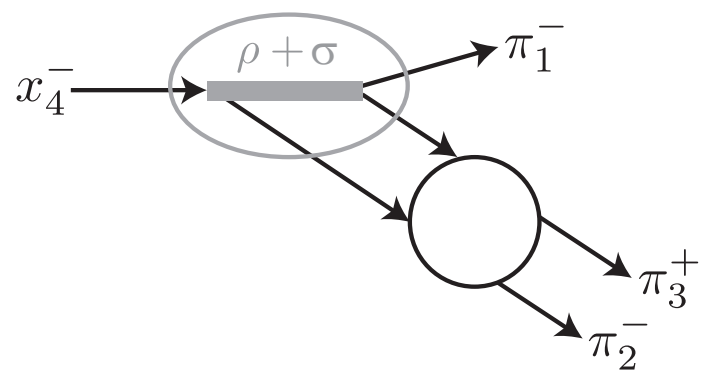

Fig. 8. This final-state interaction "generated" the Reggeons in the 23 channel and we include these in the $\rho+\sigma$ ansatz in 13 [169].

Exchange degeneracies between the leading Regge trajectories are satisfied to within roughly 10\%, and the EXD families are indicated in Fig. 9. Duality therefore leads to an important constrain that helps to reduce the number of parameters in amplitude parameterizations and improve the predictability of a fit.
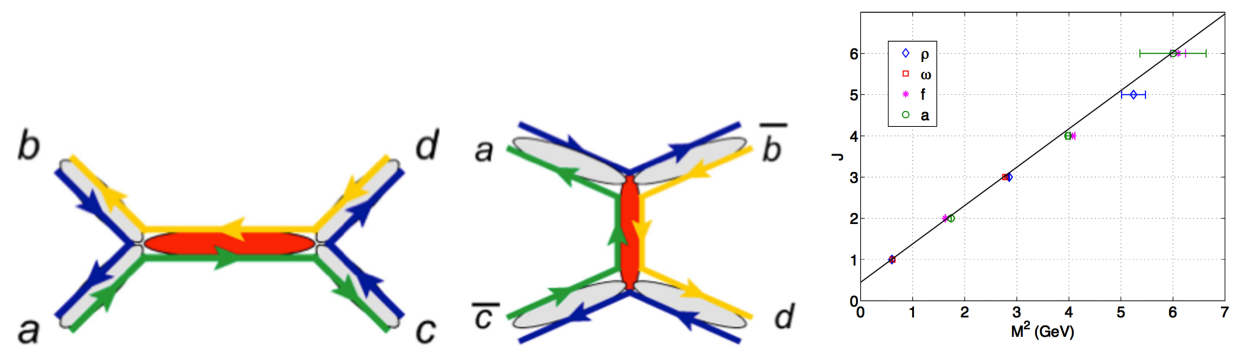

Fig. 9. Duality hypothesis as supported by the quark model. The low-energy $s$-channel amplitude (left) is related to the high-energy $t$-channel amplitude (middle). Exchange degeneracy between the $\rho, \omega, f$, and $a$ families (right).

The resonance-Reggeon duality can be extended to multiparticle production as illustrated in Figs. 10 and 11. At small scattering angle, when the center-of-mass energy of colliding hadrons is significantly above the resonance region, the reaction amplitude factorizes into a product of beam and target fragmentation sub-processes mediated by the Pomeron/Reggeon exchange as depicted in Fig. 10. With a meson or the photon as a beam and nucleon as a target, beam fragments provide the laboratory to study meson resonances, while the target fragments carry information about baryon resonances. Beam fragmentation has been the primary source of information about meson-meson phase shifts (for instance, from $\pi p \rightarrow \pi \pi p$ [170] or from $K p \rightarrow K \pi p$ [171]) and two- and three-body resonance decays. The description of the vertex representing beam-Reggeon scattering to a few meson 


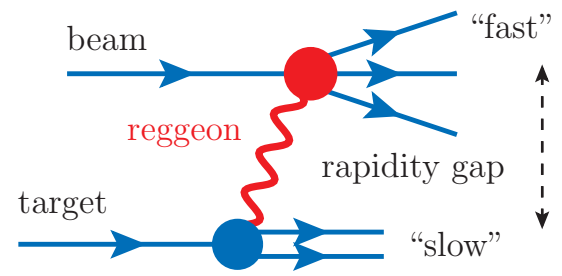

Fig. 10. Factorization in peripheral production. The upper vertex represents beam fragmentation and is described by beam plus Reggeon scattering.

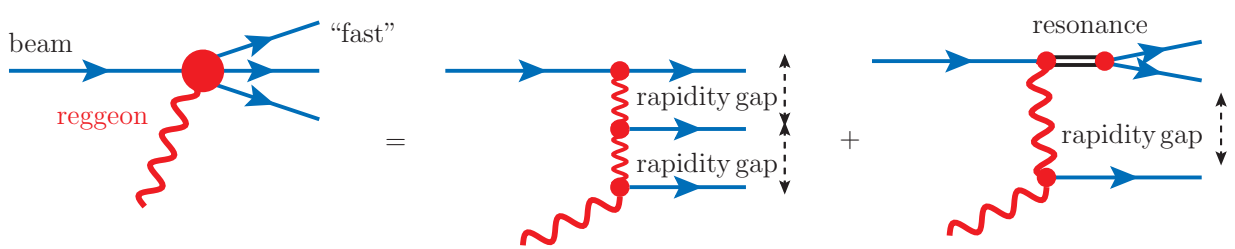

Fig. 11. Specific, non-overlapping contributions to the beam + Reggeon $\rightarrow 3$ particles amplitude.

fragments follows the principles of resonance-Reggeon duality. Again, Regge theory describes interactions between hadrons at large values of relative energy and angular momenta. It enables one to describe the bulk of the production strength outside the resonance region. The latter is parameterized in terms of a few partial waves at low masses and spins. Parameters of the low-spin partial waves can be fitted to data and self-consistency between the low-energy (resonance) and high-energy (Regge) regions is checked/enforced through finite-energy sum rules.

The leading Regge-pole dominance is an approximation, in principle valid at asymptotically large channel energies. At finite energies, the contribution from daughter trajectories and/or cuts may need to be examined on a case-by-case basis. While the Regge amplitudes turn out to provide a good qualitative description of the data even at energies as low as $2.5 \mathrm{GeV}$, a quantitative description sets in only at significantly higher energies, $c f$. Ref. [172] for a detailed discussion of $\pi N$ scattering — for a collection of earlier references, see Ref. [162]. Further research is necessary to understand which scales control the energy/momentum transfer ranges where the Regge approach is working with the accuracy necessary to meet the goals outlined above.

The cut contribution typically accounts for diffraction in the final or initial state. To test the Regge-exchange hypothesis, one can also measure semi-inclusive production: beam + target $\rightarrow$ leading particle(s) $+X$, where "leading particles" have large- $x$ [173]. This is described by some variant of Triple Regge coupling and so the "leading" part is beam + Reggeon $\rightarrow$ 
"leading particles" and so similar to the case where $X$ is a simple particle such as proton/neutron. Using arguments based on parton-hadron duality, these can be further related the partonic structure functions [174].

\section{Tools}

[R. Mitchell, D.G. Ireland]

One of the main challenges in experimental hadron spectroscopy is to determine whether or not a given data set contains evidence to support the existence of a previously unknown hadronic state (or states). The search for a new signal in experimental data consists of several steps: defining a theoretical model of the data; maximizing a likelihood function in order to fit the theoretical model to experimental data; and performing statistical tests to evaluate how well the model describes the data. The result of this process then allows one to decide whether or not a new state has been found, on the basis of model comparison.

While it is easy to list these steps, it has, in the past, not been straightforward to carry out this analysis procedure without incorporating approximations. The signals of interest are clearly not large ones (otherwise they would have already been identified!), and so we are at the stage of needing to move beyond crude approximate methods. The three steps of constructing a likelihood function based on a theoretical model, calculating the likelihood function with measured data, and evaluating the goodness-of-fit all require a set of tools that are both easy to use and contain state-of-the-art methods. Each step presents challenges:

1. How to incorporate theoretical innovations into data models (likelihood functions)?

2. How to perform efficient calculations of likelihood functions?

3. How to use statistical methods to evaluate how well theory describes data?

We now briefly summarize these issues, keeping in mind that the main framework will be a partial-wave analysis (PWA) of experimental data. In this, the key theoretical inputs are the amplitudes for participating processes. Afterwards, we list a few of the software tools that are currently being used and ideas for future collaborative code development.

\subsection{Incorporation of theoretical innovations}

The previous generation of amplitude analysis fitting tools had several undesirable features: they commonly assumed the "isobar model" with 2-body Breit-Wigner resonance decays; they were often easy to use, but 
were also a sort of black box, offering little flexibility to incorporate new amplitudes; and they were model-dependent, where the model-dependence had unquantifiable effects.

By contrast, the current generation of tools includes several desirable features: they allow more flexibility when defining amplitudes; they often force the user to explicitly code the amplitudes, but are therefore less of a black box; they incorporate state-of-the-art technology to increase fit speeds; and they allow systematic studies of model dependencies.

There are no longer experimental or technological barriers to incorporating theoretical innovations into experimental analyses. Several software packages exist that can perform fits to experimental data, using arbitrarily complicated amplitudes. An example of this is the AmpTools package ${ }^{4}$ developed at Indiana University, described further below.

\subsection{Efficient calculation of likelihood functions}

For statistical accuracy, the number of events that need to be accumulated is $\mathcal{O}\left(10^{6}\right)$. In the search for a maximum of the likelihood function, therefore, each change in the parameters of the likelihood function will require $\mathcal{O}\left(10^{6}\right)$ evaluations of the likelihood function. What is fortunate is that there are ways to make use of the implicit parallelism in this calculation that utilize the latest developments in hardware technology. The overall trend is from multi-core to many-core processors, and from parallel to massivelyparallel computing.

The most promising avenue for PWA is general purpose graphical processor unit (GPGPU) programming. Making use of the many cores on a GPU, likelihood calculations can be performed on many chunks of data at the same time. The pioneer approach of harnessing GPU parallel acceleration in PWA was performed in the framework of BES-III [175]. Presently, there are several hardware-specific programming models (CUDA, OpenCL), but the field is in a state of rapid development. Another potential game changer is Intel's Many Integrated Core (MIC) architecture (Xeon Phi).

\subsection{Statistical evaluation of results}

Having obtained an unbinned maximum likelihood to obtain estimators for any unknown parameters, the question is then "How well does the probability density function describe the data?" Unfortunately, an unbinned maximum likelihood does not provide any information that would help answer this question. Typically we (somehow) determine the " $p$-value". The $p$-value is the probability that a repeat of the experiment would have lesser agreement with the data than what we observe in our experiment.

\footnotetext{
${ }^{4}$ http://sourceforge.net/projects/amptools
} 
In a binned analysis, this is often done by determining the $\chi^{2}$ statistic. In many analyses, though, binning is not a viable option (due to high dimensions and/or low statistics). There are many methods in the statistics literature that deal with these situations. However, one must take care to choose the right tool for the job, and ensure that one can properly validate any goodness-of-fit test [176].

\subsection{Existing fitting tools and collaborative code development}

A number of software packages currently exist to aid in amplitude analysis fits. Here we mention three: AmpTools, ROOTPWA, and MadGraph.

AmpTools, mentioned above, is a set of $\mathrm{C}++$ classes that can be used for amplitude analyses. The key class is the Amplitude class, whose interface to the rest of the code is to take kinematics as input and output a complex number. The user supplies as many of these as needed. These amplitudes can be written either directly by theorists or by experimentalists in collaboration with theorists.

A new partial-wave analysis software package called ROOTPWA ${ }^{5}$ has been developed at Technische Universität München. The goal of this project is to provide a common package for the analysis of multi-body final states produced in various reactions, such as diffractive dissociation, central production, or muo-production. It includes a tool for the calculation of decay amplitudes, which is an improved implementation of the helicity-based isobar amplitude generator gamp from the PWA2000 package originally developed at BNL, augmented by scripts for automatic symmetrization and testing. The amplitude calculator can be extended to different spin formalisms and is, in principle, not limited to isobar-like decay chains. The minimization is based on MINUIT2/MIGRAD which comes as part of the ROOT toolkit. ROOTPWA is completed by an $n$-body event generator and ROOT-based visualization tools.

For systematic amplitude generation, we can mention MadGraph [177]. MadGraph, developed at the University of Illinois and at Louvain University, is a helicity amplitude generator for the tree-level Standard Model perturbation theory. It is open source and easily modifiable to include effective field theories ${ }^{6}$. Events can be generated with MadEvent, and cross sections and other observables can also be computed.

In order to make the best use of expertise to develop the best open-source software, the programming community has over the years evolved methods to make this collaboration work most efficiently. This practice is gradually being taken up in the physics research community as well. An outline of how a PWA community site might be structured is as follows:

\footnotetext{
${ }^{5}$ The software is available under GPL at http://sourceforge.net/projects/rootpwa/

${ }^{6}$ http://madgraph.phys.ucl.ac.be
} 
- Common code repository (can link to already existing sourceforge repositories) containing:

- Amplitude code;

- Data readers;

- Minimizers;

- Integrators;

- Plotters;

- Parallelization libraries;

- Exchange ideas (code snippets);

- Ecosystem of coexisting, independent codes.

\section{Concluding remarks}

The new generation of experiments in hadron physics that are currently under way or forthcoming will continue to generate complex data sets of very high quality ( $c f$. Sec. 2). Although quite involved analysis techniques are already now employed by the various experimental groups (cf., e.g., Sec. 2.3), from the theoretical side, an improved understanding of the amplitudes used is necessary to analyze and interpret the experimental results of hadron facilities in terms of resonance parameters or, at higher energies, non-perturbative quark-gluon dynamics. In addition, a full command over hadronic interactions is important to hunt for physics beyond the Standard Model. This is especially obvious when it comes to CP violation, which e.g. in the decays of heavy mesons becomes visible only via the interference with strong phases.

The key theoretical developments of the last decade were in the realm of:

- dispersion theory, especially for low- and medium-energy $\pi \pi$ interactions (up to $\sqrt{s}$ of about $1.4 \mathrm{GeV}$ ) as well as pion rescattering in few-body final-state interactions (cf. Sec. 3.1),

- effective Lagrangian approaches, mainly for meson-baryon systems in the resonance region (cf. Sec. 3.2),

- Regge theory, which is essential at high energies and for reactions in peripheral kinematics (cf. Sec. 3.3).

Besides further improvements within the three approaches themselves, the central goals for the future concern the merging of the different methods and the reliable estimate of theoretical uncertainties. For the former, it will be crucial to identify proper matching criteria in the kinematic regions of overlap, for the latter to find ways to systematically monitor the accuracy of approximations made. 
The aim of this document is to initiate or intensify the discussion on the methodology and tools needed to achieve these goals. We expect this discussion to continue through a series of workshops and schools that are planned for the near future. We hope these will lead to the development of state-of-the-art analysis tools that will become available to practitioners of amplitude analysis techniques in the interpretation of experimental data.

\section{REFERENCES}

[1] K.A. Olive et al. [Particle Data Group], Chin. Phys. C38, 090001 (2014).

[2] J.J. Dudek et al. [Hadron Spectrum Collaboration], Phys. Rev. D88, 094505 (2013) [arXiv:1309.2608 [hep-lat]].

[3] J.J. Dudek et al., Phys. Rev. D82, 034508 (2010) [[arXiv: 1004.4930 [hep-ph]].

[4] J.J. Dudek et al., Phys. Rev. D83, 111502 (2011) [arXiv:1102.4299 [hep-lat]].

[5] S. Dürr et al., Science 322, 1224 (2008) [arXiv:0906.3599 [hep-lat]].

[6] L. Liu et al. [Hadron Spectrum Collaboration], J. High Energy Phys. 1207, 126 (2012) [arXiv:1204.5425 [hep-ph]].

[7] P. Guo et al., Phys. Rev. D78, 056003 (2008) [arXiv:0807.2721 [hep-ph]].

[8] R.G. Edwards, J.J. Dudek, D.G. Richards, S.J. Wallace, Phys. Rev. D84, 074508 (2011) [arXiv:1104.5152 [hep-ph]].

[9] R.G. Edwards et al. [Hadron Spectrum Collaboration], Phys. Rev. D87, 054506 (2013) [arXiv:1212.5236 [hep-ph]].

[10] J.M. Bulava et al., Phys. Rev. D79, 034505 (2009) [arXiv:0901.0027 [hep-lat]].

[11] C. Alexandrou, T. Korzec, G. Koutsou, T. Leontiou, Phys. Rev. D89, 034502 (2014) [arXiv:1302.4410 [hep-lat]].

[12] G.P. Engel et al. [BGR Collaboration], Phys. Rev. D87, 074504 (2013) [arXiv:1301.4318 [hep-lat]].

[13] M.S. Mahbub et al. [CSSM Lattice Collaboration], Phys. Rev. D87, 011501 (2013) [arXiv:1209.0240 [hep-lat]].

[14] J.J. Dudek, R.G. Edwards, Phys. Rev. D85, 054016 (2012) [arXiv:1201.2349 [hep-ph]].

[15] C.J. Morningstar, M.J. Peardon, Phys. Rev. D60, 034509 (1999) [arXiv: hep-lat/9901004].

[16] Y. Chen et al., Phys. Rev. D73, 014516 (2006) [arXiv:hep-lat/0510074].

[17] M. Lüscher, Nucl. Phys. B354, 531 (1991).

[18] K. Rummukainen, S.A. Gottlieb, Nucl. Phys. B450, 397 (1995) [arXiv:hep-lat/9503028]. 
[19] C.H. Kim, C.T. Sachrajda, S.R. Sharpe, Nucl. Phys. B727, 218 (2005) [arXiv:hep-lat/0507006].

[20] N.H. Christ, C.H. Kim, T. Yamazaki, Phys. Rev. D72, 114506 (2005) [arXiv:hep-lat/0507009].

[21] X. Feng, K. Jansen, D.B. Renner, Phys. Rev. D83, 094505 (2011) [arXiv:1011.5288 [hep-lat]].

[22] C.B. Lang, D. Mohler, S. Prelovsek, M. Vidmar, Phys. Rev. D84, 054503 (2011) [Erratum ibid. D89, 059903 (2014)] [arXiv:1105.5636 [hep-lat]].

[23] M. Döring, U.-G. Meißner, E. Oset, A. Rusetsky, Eur. Phys. J. A47, 139 (2011) [arXiv:1107.3988 [hep-lat]].

[24] M. Döring, U.-G. Meißner, E. Oset, A. Rusetsky, Eur. Phys. J. A48, 114 (2012) [arXiv:1205.4838 [hep-lat]].

[25] M. Göckeler et al., Phys. Rev. D86, 094513 (2012) [arXiv:1206.4141 [hep-lat]].

[26] J.J. Dudek et al. [Hadron Spectrum Collaboration], Phys. Rev. Lett. 113, 182001 (2014) [arXiv:1406.4158 [hep-ph]].

[27] M. Lage, U.-G. Meißner, A. Rusetsky, Phys. Lett. B681, 439 (2009) [arXiv:0905.0069 [hep-lat]].

[28] V. Bernard, M. Lage, U.-G. Meißner, A. Rusetsky, J. High Energy Phys. 1101, 019 (2011) [arXiv:1010.6018 [hep-lat]].

[29] R.A. Briceno, Z. Davoudi, Phys. Rev. D88, 094507 (2013) [arXiv:1204.1110 [hep-lat]].

[30] M.T. Hansen, S.R. Sharpe, Phys. Rev. D86, 016007 (2012) [arXiv:1204.0826 [hep-lat]].

[31] P. Guo, J.J. Dudek, R. Edwards, A.P. Szczepaniak, Phys. Rev. D88, 014501 (2013) [arXiv: 1211.0929 [hep-lat]].

[32] J.J. Dudek, R.G. Edwards, C.E. Thomas, Phys. Rev. D87, 034505 (2013) [arXiv:1212.0830 [hep-ph]].

[33] C. Pelissier, A. Alexandru, Phys. Rev. D87, 014503 (2013) [arXiv:1211.0092 [hep-lat]].

[34] S. Aoki et al. [CS Collaboration], Phys. Rev. D84, 094505 (2011) [arXiv:1106.5365 [hep-lat]].

[35] J. Frison et al. [Budapest-Marseille-Wuppertal Collaboration], PoS LATTICE2010, 139 (2010) [arXiv:1011.3413 [hep-lat]].

[36] M. Göckeler et al. [QCDSF Collaboration], PoS LATTICE2008, 136 (2008) [arXiv:0810.5337 [hep-lat]].

[37] K. Polejaeva, A. Rusetsky, Eur. Phys. J. A48, 67 (2012) [arXiv:1203.1241 [hep-lat]].

[38] R.A. Briceno, Z. Davoudi, Phys. Rev. D87, 094507 (2013) [arXiv:1212.3398 [hep-lat]].

[39] S. Kreuzer, H.W. Grießhammer, Eur. Phys. J. A48, 93 (2012) [arXiv:1205.0277 [nucl-th]]. 
[40] M.T. Hansen, S.R. Sharpe, PoS LATTICE2013, 221 (2014) [arXiv: 1311.4848 [hep-lat]].

[41] P. Abbon et al. [COMPASS Collaboration], Nucl. Instrum. Methods A577, 455 (2007) [arXiv: hep-ex/0703049].

[42] P. Abbon et al. [COMPASS Collaboration], arXiv:1410.1797 [physics.ins-det].

[43] M. Alekseev et al. [COMPASS Collaboration], Phys. Rev. Lett. 104, 241803 (2010) [arXiv:0910.5842 [hep-ex]].

[44] C. Adolph et al. [Compass Collaboration], arXiv:1408.4286 [hep-ex].

[45] G.F. Chew, M.L. Goldberger, F.E. Low, Y. Nambu, Phys. Rev. 106, 1345 (1957).

[46] J.M. Gerard, J. Weyers, Eur. Phys. J. C7, 1 (1999) [arXiv:hep-ph/9711469].

[47] J.M. Gerard, J. Pestieau, J. Weyers, Phys. Lett. B436, 363 (1998) [arXiv:hep-ph/9803328].

[48] D.M. Asner et al., Int. J. Mod. Phys. A24, S1 (2009) [arXiv:0809.1869 [hep-ex]].

[49] R.R. Akhmetshin et al. [CMD2 Collaboration], Phys. Lett. B466, 392 (1999) [arXiv:hep-ex/9904024].

[50] R.R. Akhmetshin et al. [CMD-2 Collaboration], Phys. Lett. B595, 101 (2004) [arXiv:hep-ex/0404019].

[51] K.W. Edwards et al. [CLEO Collaboration], Phys. Rev. D61, 072003 (2000) [arXiv:hep-ex/9908024].

[52] V.M. Aulchenko et al. [KEDR Collaboration], Phys. Lett. B573, 63 (2003) [arXiv: hep-ex/0306050].

[53] V.V. Anashin et al. [KEDR Collaboration], Phys. Lett. B685, 134 (2010) [arXiv:0912.1082 [hep-ex]].

[54] V.V. Anashin et al., Phys. Lett. B711, 280 (2012) [arXiv:1109.4215 [hep-ex]].

[55] V.V. Anashin et al., Phys. Lett. B711, 292 (2012) [arXiv:1109.4205 [hep-ex]].

[56] V.V. Anashin et al. [KEDR Collaboration], Phys. Lett. B686, 84 (2010) [arXiv:0909.5545 [hep-ex]].

[57] S.I. Eidelman et al. [KEDR Collaboration], Nucl. Phys. Proc. Suppl. 218, 155 (2011).

[58] V.V. Anashin et al. [KEDR Collaboration], Phys. Lett. B703, 543 (2011) [arXiv:1107.2824 [hep-ex]].

[59] A.A. Alves Jr. et al. [LHCb Collaboration], JINST 3, S08005 (2008).

[60] R. Aaij et al. [LHCb Collaboration], Phys. Lett. B694, 209 (2010) [arXiv: 1009.2731 [hep-ex]].

[61] R. Aaij et al. [LHCb Collaboration], LHCb-CONF-2010-013, CERN-LHCb-CONF-2010-013. 
[62] R. Aaij et al. [LHCb Collaboration], Nucl. Phys. B871, 1 (2013) [arXiv:1302.2864 [hep-ex]].

[63] C.W. Salgado, D.P. Weygand, Phys. Rep. 537, 1 (2014) [arXiv:1310.7498 [nucl-ex]].

[64] S.-U. Chung, CERN-71-08.

[65] I.J.R. Aitchison, J.J. Brehm, Phys. Lett. B84, 349 (1979).

[66] I.J.R. Aitchison, J.J. Brehm, Phys. Rev. D20, 1131 (1979).

[67] K.L. Au, D. Morgan, M.R. Pennington, Phys. Rev. D35, 1633 (1987).

[68] V.V. Anisovich, A.V. Sarantsev, Eur. Phys. J. A16, 229 (2003) [arXiv: hep-ph/0204328].

[69] S.-U. Chung, T.L. Trueman, Phys. Rev. D11, 633 (1975).

[70] S. Neubert, Ph.D. Thesis, Techn. Univ. München, 2012.

[71] G.F. Chew, M.L. Goldberger, F.E. Low, Y. Nambu, Phys. Rev. 106, 1337 (1957).

[72] A.M. Sandorfi, S. Hoblit, H. Kamano, T.-S.H. Lee, J. Phys. G 38, 053001 (2011) [arXiv:1010.4555 [nucl-th]].

[73] Y. Wunderlich, R. Beck, L. Tiator, arXiv:1312.0245 [nucl-th].

[74] R.L. Workman et al., Eur. Phys. J. A47, 143 (2011) [arXiv:1102.4897 [nucl-th]].

[75] Exciting Baryons: Design, Analysis of Complete Experiments for Meson Photoproduction, Trento, ECT*, June 30-July 4, 2014.

[76] L. Tiator, Bled Workshops in Physics. Vol. 13, No. 1 [arXiv:1211.3927 [nucl-th]].

[77] R.A. Arndt, W.J. Briscoe, I.I. Strakovsky, R.L. Workman, Phys. Rev. C74, 045205 (2006) [arXiv:nucl-th/0605082].

[78] R.L. Workman, M.W. Paris, W.J. Briscoe, I.I. Strakovsky, Phys. Rev. C86, 015202 (2012) [arXiv: 1202.0845 [hep-ph]].

[79] D. Drechsel, S.S. Kamalov, L. Tiator, Eur. Phys. J. A34, 69 (2007) [arXiv:0710.0306 [nucl-th]].

[80] A.V. Anisovich et al., Eur. Phys. J. A49, 121 (2013) [arXiv:1306.5126 [hep-ph]].

[81] J. Hartmann et al., Phys. Rev. Lett. 113, 062001 (2014) [arXiv:1407.2163 [nucl-ex]].

[82] "Workshop with Secondary Hadron Beams in the 21st Century", http://gwdac.phys.gwu .edu/ igor/Ashburn2012/Home.html, GWU/VSTC, April 7, 2012.

[83] A.D. Martin, T.D. Spearman, Elementary Particle Theory, North Holland Publishing Co., Amsterdam 1970.

[84] M.L. Perl, High Energy Hadron Physics, John Wiley \& Sons., New York 1975. 
[85] M.L. Goldberger, K.M. Watson, Collision Theory, John Wiley \& Sons., New York 1965.

[86] J. Gasser, G. Wanders, Eur. Phys. J. C10, 159 (1999) [arXiv:hep-ph/9903443].

[87] G. Wanders, Eur. Phys. J. C17, 323 (2000) [arXiv:hep-ph/0005042].

[88] B. Ananthanarayan, G. Colangelo, J. Gasser, H. Leutwyler, Phys. Rep. 353, 207 (2001) [arXiv:hep-ph/0005297].

[89] P. Büttiker, S. Descotes-Genon, B. Moussallam, Eur. Phys. J. C33, 409 (2004) [arXiv:hep-ph/0310283].

[90] I. Caprini, G. Colangelo, H. Leutwyler, Phys. Rev. Lett. 96, 132001 (2006) [arXiv:hep-ph/0512364].

[91] R. García-Martín, R. Kamiński, J.R. Peláez, J. Ruiz de Elvira, Phys. Rev. Lett. 107, 072001 (2011) [arXiv:1107.1635 [hep-ph]].

[92] S. Descotes-Genon, B. Moussallam, Eur. Phys. J. C48, 553 (2006) [arXiv:hep-ph/0607133].

[93] P. Hoyer, J. Kwieciński, Nucl. Phys. B60, 26 (1973).

[94] S.M. Roy, Phys. Lett. B36, 353 (1971).

[95] F. Steiner, Fortsch. Phys. 19, 115 (1971).

[96] G.E. Hite, F. Steiner, Nuovo Cim. A18, 237 (1973).

[97] G. Colangelo, J. Gasser, H. Leutwyler, Nucl. Phys. B603, 125 (2001) [arXiv:hep-ph/0103088].

[98] R. Kamiński, L. Lesniak, B. Loiseau, Phys. Lett. B551, 241 (2003) [arXiv:hep-ph/0210334].

[99] J.R. Peláez, F.J. Ynduráin, Phys. Rev. D71, 074016 (2005) [arXiv:hep-ph/0411334].

[100] R. García-Martín et al., Phys. Rev. D83, 074004 (2011) [arXiv:1102.2183 [hep-ph]].

[101] J.A. Oller, L. Roca, C. Schat, Phys. Lett. B659, 201 (2008) [arXiv:0708.1659 [hep-ph]].

[102] M. Hoferichter, D.R. Phillips, C. Schat, Eur. Phys. J. C71, 1743 (2011) [arXiv:1106.4147 [hep-ph]].

[103] R. García-Martín, B. Moussallam, Eur. Phys. J. C70, 155 (2010) [arXiv:1006.5373 [hep-ph]].

[104] B. Moussallam, Eur. Phys. J. C71, 1814 (2011) [arXiv:1110.6074 [hep-ph]].

[105] C. Ditsche, M. Hoferichter, B. Kubis, U.-G. Meißner, J. High Energy Phys. 1206, 043 (2012) [arXiv:1203.4758 [hep-ph]].

[106] M. Froissart, Phys. Rev. 123, 1053 (1961).

[107] A. Martin, Phys. Rev. 129, 1432 (1963).

[108] J.R. Peláez, F.J. Ynduráin, Phys. Rev. D69, 114001 (2004) [arXiv:hep-ph/0312187]. 
[109] F. Halzen, K. Igi, M. Ishida, C.S. Kim, Phys. Rev. D85, 074020 (2012) [arXiv:1110.1479 [hep-ph]].

[110] I. Caprini, G. Colangelo, H. Leutwyler, Eur. Phys. J. C72, 1860 (2012) [arXiv:1111.7160 [hep-ph]].

[111] K.M. Watson, Phys. Rev. 95, 228 (1954).

[112] E. Fermi, Nuovo Cim. 2S1, 17 (1955) [Riv. Nuovo Cim. 31, 1 (2008)].

[113] R. Omnès, Nuovo Cim. 8, 316 (1958).

[114] N.I. Muskhelishvili, Singular Integral Equations, Wolters-Noordhoff Publishing, Groningen 1953.

[115] M. Gorchtein, P. Guo, A.P. Szczepaniak, Phys. Rev. C86, 015205 (2012) [arXiv:1102.5558 [nucl-th]].

[116] C. Hanhart, Phys. Lett. B715, 170 (2012) [arXiv:1203.6839 [hep-ph]].

[117] B. Ananthanarayan, I. Caprini, I. Sentitemsu Imsong, Eur. Phys. J. A47, 147 (2011) [arXiv: 1108.0284 [hep-ph]].

[118] G. Abbas et al., Eur. Phys. J. A44, 175 (2010) [arXiv:0912.2831 [hep-ph]].

[119] A.P. Szczepaniak, P. Guo, M. Battaglieri, R. De Vita, Phys. Rev. D82, 036006 (2010) [arXiv:1005.5562 [hep-ph]].

[120] G. Abbas, B. Ananthanarayan, I. Caprini, I. Sentitemsu Imsong, Phys. Rev. D82, 094018 (2010) [arXiv: 1008.0925 [hep-ph]].

[121] P. Guo, R. Mitchell, A.P. Szczepaniak, Phys. Rev. D82, 094002 (2010) [arXiv:1006.4371 [hep-ph]].

[122] M. Hoferichter et al., Eur. Phys. J. C74, 3180 (2014) [arXiv:1410.4691 [hep-ph]].

[123] N.N. Khuri, S.B. Treiman, Phys. Rev. 119, 1115 (1960).

[124] F. Stollenwerk et al., Phys. Lett. B707, 184 (2012) [arXiv:1108.2419 [nucl-th]].

[125] M. Hoferichter, B. Kubis, D. Sakkas, Phys. Rev. D86, 116009 (2012) [arXiv:1210.6793 [hep-ph]].

[126] J. Kambor, C. Wiesendanger, D. Wyler, Nucl. Phys. B465, 215 (1996) [arXiv:hep-ph/9509374].

[127] G. Colangelo, S. Lanz, E. Passemar, PoS CD09, 047 (2009) [arXiv:0910.0765 [hep-ph]].

[128] S.P. Schneider, B. Kubis, C. Ditsche, J. High Energy Phys. 1102, 028 (2011) [arXiv:1010.3946 [hep-ph]].

[129] F. Niecknig, B. Kubis, S.P. Schneider, Eur. Phys. J. C72, 2014 (2012) [arXiv:1203.2501 [hep-ph]].

[130] I.V. Danilkin et al., arXiv:1409.7708 [hep-ph].

[131] J.F. Donoghue, J. Gasser, H. Leutwyler, Nucl. Phys. B343, 341 (1990).

[132] B. Moussallam, Eur. Phys. J. C14, 111 (2000) [arXiv:hep-ph/9909292]. 
[133] S. Descotes-Genon, J. High Energy Phys. 0103, 002 (2001) [arXiv:hep-ph/0012221].

[134] M. Hoferichter, C. Ditsche, B. Kubis, U.-G. Meißner, J. High Energy Phys. 1206, 063 (2012) [arXiv:1204.6251 [hep-ph]].

[135] J.T. Daub et al., J. High Energy Phys. 1301, 179 (2013) [arXiv:1212.4408 [hep-ph]].

[136] M. Jamin, J.A. Oller, A. Pich, Nucl. Phys. B622, 279 (2002) [arXiv:hep-ph/0110193].

[137] M. Döring, U.-G. Meißner, W. Wang, J. High Energy Phys. 1310, 011 (2013) [arXiv:1307.0947 [hep-ph]].

[138] S. Ceci et al., Phys. Rev. C84, 015205 (2011) [arXiv:1104.3490 [nucl-th]].

[139] H. Kamano, S.X. Nakamura, T.-S.H. Lee, T. Sato, Phys. Rev. C88, 035209 (2013) [arXiv:1305.4351 [nucl-th]].

[140] D. Rönchen et al., Eur. Phys. J. A50, 101 (2014) [arXiv:1401.0634 [nucl-th]].

[141] D. Rönchen et al., Eur. Phys. J. A49, 44 (2013) [arXiv:1211.6998 [nucl-th]].

[142] L. Tiator et al., Phys. Rev. C82, 055203 (2010) [arXiv:1007.2126 [nucl-th]].

[143] C. Schutz, J.W. Durso, K. Holinde, J. Speth, Phys. Rev. C49, 2671 (1994).

[144] R. Aaron, R.D. Amado, J.E. Young, Phys. Rev. 174, 2022 (1968).

[145] H. Kamano, S.X. Nakamura, T.-S.H. Lee, T. Sato, Phys. Rev. D84, 114019 (2011) [arXiv:1106.4523 [hep-ph]].

[146] P.C. Magalhães et al., Phys. Rev. D84, 094001 (2011) [arXiv:1105.5120 [hep-ph]].

[147] A.V. Anisovich et al., Eur. Phys. J. A48, 15 (2012) [arXiv:1112.4937 [hep-ph]].

[148] A.V. Anisovich et al., Eur. Phys. J. A48, 88 (2012) [arXiv:1205.2255 [nucl-th]].

[149] M. Shrestha, D.M. Manley, Phys. Rev. C86, 055203 (2012) [arXiv:1208.2710 [hep-ph]].

[150] M. Batinic, S. Ceci, A. Švarc, B. Zauner, Phys. Rev. C82, 038203 (2010).

[151] V. Shklyar, H. Lenske, U. Mosel, G. Penner, Phys. Rev. C71, 055206 (2005) [Erratum ibid. C72, 019903 (2005)] [arXiv:nucl-th/0412029].

[152] V. Shklyar, H. Lenske, U. Mosel, Phys. Rev. C87, 015201 (2013) [arXiv:1206.5414 [nucl-th]].

[153] I.G. Aznauryan et al., Int. J. Mod. Phys. E22, 1330015 (2013) [arXiv:1212.4891 [nucl-th]].

[154] I.G. Aznauryan et al., Phys. Rev. C72, 045201 (2005) [arXiv:hep-ph/0508057]. 
[155] A. Anisovich, E. Klempt, A. Sarantsev, U. Thoma, Eur. Phys. J. A24, 111 (2005) [arXiv: hep-ph/0407211].

[156] E. Gutz et al. [CBELSA/TAPS Collaboration], Eur. Phys. J. A50, 74 (2014) [arXiv:1402.4125 [nucl-ex]].

[157] V. Shklyar, H. Lenske, U. Mosel, arXiv:1409.7920 [nucl-th].

[158] H. Kamano et al., Phys. Rev. C79, 025206 (2009) [arXiv:0807.2273 [nucl-th]].

[159] D.M. Manley, R.A. Arndt, Y. Goradia, V.L. Teplitz, Phys. Rev. D30, 904 (1984).

[160] R.L. Workman, R.A. Arndt, Phys. Rev. D45, 1789 (1992).

[161] A.V. Anisovich et al., Eur. Phys. J. A49, 158 (2013) [arXiv:1310.3610 [nucl-ex]].

[162] P.D.B. Collins, Phys. Rep. 1, 103 (1971).

[163] P.D.B. Collins, An Introduction to Regge Theory and High-energy Physics, Cambridge UP, 1977.

[164] R. Dolen, D. Horn, C. Schmid, Phys. Rev. Lett. 19, 402 (1967).

[165] R. Dolen, D. Horn, C. Schmid, Phys. Rev. 166, 1768 (1968).

[166] J. Mandula, J. Weyers, G. Zweig, Annu. Rev. Nucl. Part. Sci. 20, 289 (1970).

[167] R.J.N. Phillips, D.P. Roy, Rep. Prog. Phys. 37, 1035 (1974).

[168] J. Ruiz de Elvira, J.R. Peláez, M.R. Pennington, D.J. Wilson, Phys. Rev. D84, 096006 (2011) [arXiv: 1009.6204 [hep-ph]].

[169] G.C. Fox [CMD2 Collaboration], Planning a New Generation of Multiparticle Phase Shift Analyses in Peripheral Reactions grids.ucs.indiana.edu/ptliupages/publications/gluonic_gcf.pdf Proceedings of Jefferson Laboratory Workshop on Gluonic Excitations Newport News, May 16, 2003.

[170] G. Grayer et al., Nucl. Phys. B75, 189 (1974).

[171] D. Aston et al., Nucl. Phys. B296, 493 (1988).

[172] F. Huang et al., Eur. Phys. J. A40, 77 (2009) [arXiv:0810.2680 [hep-ph]].

[173] A.V. Barnes et al., Nucl. Phys. B145, 45 (1978).

[174] J.D. Bjorken, J.B. Kogut, Phys. Rev. D8, 1341 (1973).

[175] N. Berger, B.J. Liu, J.K. Wang, J. Phys. Conf. Ser. 219, 042031 (2010).

[176] M. Williams, JINST 5, P09004 (2010) [arXiv:1006.3019 [hep-ex]].

[177] J. Alwall et al., J. High Energy Phys. 1106, 128 (2011) [arXiv:1106.0522 [hep-ph]]. 